\title{
Division dans l'anneau des séries formelles à croissance contrôlée. Applications
}

\author{
par \\ Augustin Mouze (Lille)
}

\begin{abstract}
We consider subrings $A$ of the ring of formal power series. They are defined by growth conditions on coefficients such as, for instance, Gevrey conditions. We prove a Weierstrass-Hironaka division theorem for such subrings. Moreover, given an ideal $\mathcal{I}$ of $A$ and a series $f$ in $A$ we prove the existence in $A$ of a unique remainder $r$ modulo $\mathcal{I}$. As a consequence, we get a new proof of the noetherianity of $A$.
\end{abstract}

Introduction. Dans toute la suite, $M=\left\{M_{n}\right\}_{n \in \mathbb{N}}$ désigne une suite de réels positifs qui vérifie les propriétés

$$
\begin{gathered}
M_{0}=1 \text { et }\left\{M_{n}\right\}_{n \in \mathbb{N}} \text { est logarithmiquement convexe, } \\
\exists C>0 \text { tel que } M_{n+1} \leq C^{n+1} M_{n} .
\end{gathered}
$$

La condition $\left(\mathrm{H}_{1}\right)$ équivaut à la croissance de la suite $\left\{M_{n+1} / M_{n}\right\}_{n \in \mathbb{N}}$ et entraîne l'inégalité $M_{j} M_{k} \leq M_{j+k}$ pour tous $j, k$ entiers.

On note aussi, dans la suite, pour tout entier fixé $k, M_{+k}$ la suite $\left\{M_{n+k}\right\}_{n \in \mathbb{N}}, M_{-k}$ la suite $\left\{M_{n-k}\right\}_{n \in \mathbb{N}, n \geq k}$ et $M^{(k)}$ la suite $\left\{M_{k n}\right\}_{n \in \mathbb{N}}$.

Soit $\mathbb{K}=\mathbb{R}$ ou $\mathbb{C}$. On désigne alors par $\mathbb{K}[[X]]$ l'anneau des séries formelles en $s$ variables à coefficients dans le corps $\mathbb{K}$. Pour tout multi-indice $J$ de $\mathbb{N}^{s}$, on note $X^{J}=X_{1}^{j_{1}} \ldots X_{s}^{j_{s}}$ et $|J|=j_{1}+\ldots+j_{s}$. Soit $r=\left(r_{1}, \ldots, r_{s}\right)$ un poly-rayon de $\mathbb{R}_{+}^{* s}$. On note

$$
\mathbb{K}[[X]](M, r)=\left\{f \in \mathbb{K}[[X]]: f=\sum_{J \in \mathbb{N}^{s}} f_{J} X^{J}, \sum_{j=0}^{\infty} \sum_{\substack{J \in \mathbb{N}^{s} \\|J|=j}} \frac{\left|f_{J}\right|}{M_{j}} r^{J}<\infty\right\}
$$

et

$$
\mathbb{K}[[X]](M)=\bigcup_{r} \mathbb{K}[[X]](M, r) .
$$

On dit que l'anneau $\mathbb{K}[[X]](M)$ est un anneau de séries formelles à croissance contrôlée. C'est une limite inductive d'espaces de Banach. Si $M=1$, c'est-

2000 Mathematics Subject Classification: 13F25, 13J15, 16P40, 32A05. 
à-dire si $M_{n}=1$ pour tout entier $n$, alors $\mathbb{K}[[X]](1)$ n'est autre que $\mathbb{K}\{X\}$, l'anneau des séries convergentes.

Afin d'étudier les propriétés de $\mathbb{K}[[X]](M)$, on se propose d'établir, dans cet anneau, un théorème de division du type Weierstrass par plusieurs séries. Une version du théorème de préparation de Weierstrass et de division par une seule série a déjà été donnée par J. Chaumat et A.-M. Chollet [3]. On retrouve leur résultat ici. Il s'agit des théorèmes 3.1 et 3.2. Dans le même article, les auteurs montrent que, sous l'hypothèse $\left(\mathrm{H}_{1}\right)$, la condition $\left(\mathrm{H}_{2}\right)$ est nécessaire et suffisante pour que l'anneau $\mathbb{K}[[X]](M)$ soit noethérien. On notera que les énoncés font appel à une notion de $p$-régularité plus restrictive que la notion de régularité d'ordre $p$ classique dans $\mathbb{K}[[X]]$ et $\mathbb{K}\{X\}$. On sait $[3]$, en effet, que la division dans $\mathbb{K}[[X]](M)$ par une série régulière d'ordre $p$ met, en général, en évidence une perte de régularité sur la croissance des coefficients du quotient et du reste dans cette division. Ce phénomène a été noté également par M. A. Zurro [8] dans le cadre de la division par plusieurs séries formelles, au sens d'Hironaka [1]; en effet, dans [8], on trouve des énoncés avec "perte de régularité" concernant les anneaux à croissance Gevrey $\left(M_{n}=n !^{\alpha}, \alpha \in \mathbb{R}\right)$.

0. Présentation des principaux résultats. Dans ce papier, on établit tout d'abord un théorème de division au sens d'Hironaka dans $\mathbb{K}[[X]](M)$.

0.1. Notations. Dans toute la suite, pour tout $f \in \mathbb{K}[[X]]$, on note $f_{J}$ l'élément de $\mathbb{K}$ défini par la formule $f(X)=\sum_{J \in \mathbb{N}^{s}} f_{J} X^{J}$. On définit alors l'ensemble d'exposants

$$
\operatorname{Exp}_{X}(f)=\left\{J \in \mathbb{N}^{s}: f_{J} \neq 0\right\} .
$$

Soient $p$ multi-indices de $\mathbb{N}^{s}, E_{1}, \ldots, E_{p}$. On note

$$
\Delta=\bigcup_{i=1}^{p}\left(E_{i}+\mathbb{N}^{s}\right) .
$$

On choisit alors une partition $\Delta=\Delta_{1} \cup \ldots \cup \Delta_{p}$ de $\Delta$ telle que, pour tout $i=1, \ldots, p, \Delta_{i} \subset E_{i}+\mathbb{N}^{s}$. Par exemple, on pose $\Delta_{1}=E_{1}+\mathbb{N}^{s}$, $\Delta_{2}=\left(E_{2}+\mathbb{N}^{s}\right)-\Delta_{1}, \ldots, \Delta_{i}=\left(E_{i}+\mathbb{N}^{s}\right)-\left(\Delta_{1} \cup \ldots \cup \Delta_{i-1}\right)$. Dans toute la suite, on dira que $\mathbb{N}^{s}=\Delta_{1} \cup \ldots \cup \Delta_{p} \cup\left(\mathbb{N}^{s}-\Delta\right)$ est la partition de $\mathbb{N}^{s}$ associée aux $E_{i}, i=1, \ldots, p$.

Avec ces notations, on a le résultat suivant, prouvé en 2.4.

0.2. ThÉorème (de $L$-division). Soient $f_{1}, \ldots, f_{p}$ dans $\mathbb{K}[[X]](M)$. Soient $L$ une forme linéaire sur $\mathbb{R}^{s}$ à coefficients strictement positifs et $E_{1}, \ldots, E_{p}$ des multi-indices de $\mathbb{N}^{s}$ tels que, si on note $f_{i}=\sum_{J \in \mathbb{N}^{s}}\left(f_{i}\right)_{J} X^{J}$, on ait 
(i) $\left(f_{i}\right)_{J}=0$ pour tout $J \in \mathbb{N}^{s},|J|<\left|E_{i}\right|$,

(ii) $\left(f_{i}\right)_{E_{i}} \neq 0$,

(iii) $\left(f_{i}\right)_{J}=0$ pour tout $J \in \mathbb{N}^{s}, J \neq E_{i}$ et $L(J) \leq L\left(E_{i}\right)$.

Alors, pour tout élément $g$ de $\mathbb{K}[[X]](M)$, il existe des uniques séries $g_{1}, \ldots$ $\ldots, g_{p}, h$ de $\mathbb{K}[[X]](M)$ telles que l'on ait

$$
\begin{gathered}
g=\sum_{i=1}^{p} f_{i} g_{i}+h, \\
\operatorname{Exp}_{X}\left(g_{i}(X) X^{E_{i}}\right) \subset \Delta_{i}, \quad i=1, \ldots, p, \\
\sum_{b=0}^{\infty} \sum_{\substack{B \in \mathbb{N}^{s} \\
|B|=b}} h_{B} X^{B} \quad \text { avec } h_{B}=0 \text { pour } B \in \Delta,
\end{gathered}
$$

où $\mathbb{N}^{s}=\Delta_{1} \cup \ldots \cup \Delta_{p} \cup\left(\mathbb{N}^{s}-\Delta\right)$ est la partition de $\mathbb{N}^{s}$ associée aux $E_{i}, 1 \leq i \leq p$. De plus, $\left(g_{1}, \ldots, g_{p} ; h\right)$ est donné par un opérateur linéaire continu.

Les conditions (i)-(iii) généralisent la notion de p-régularité de [3]. Elles ne sont pas restrictives. On pourra s'en convaincre à la lecture du paragraphe 2 .

Pour établir ce théorème, on adapte une preuve, donnée par J. Briançon, d'un théorème de division dans $\mathbb{K}\{X\}$, par perturbation d'un épimorphisme [2].

On applique ensuite ce théorème au problème de division par un idéal. On considère un idéal de $\mathbb{K}[[X]](M)$. Peut-on associer, à toute série de $\mathbb{K}[[X]](M)$, un reste unique, dans $\mathbb{K}[[X]](M)$, modulo cet idéal? On apporte une réponse affirmative à ce problème. Pour cela, on construit une famille génératrice de l'idéal en s'inspirant des bases de Groebner d'un idéal polynômial [4]. On a alors l'énoncé suivant qui sera précisé et prouvé dans le paragraphe 4 .

0.3. ThÉorÈme. Soient $f_{1}, \ldots, f_{p}$ des éléments de $\mathbb{K}[[X]](M)$. On note $\mathcal{I}_{M}$ l'idéal engendré par ces éléments sur $\mathbb{K}[[X]](M)$. Il existe alors une famille géneratrice $G=\left(g_{1}, \ldots, g_{k}\right)$ pour $\mathcal{I}_{M}$ telle que, pour tout $i=1, \ldots, k$, $g_{i}$ appartienne à $\mathbb{K}[[X]](M)$, et telle que, pour tout $g$ dans $\mathbb{K}[[X]](M)$, on puisse écrire $g=\sum_{i=1}^{k} h_{i} g_{i}+h_{0}$ avec, pour tout $i=0, \ldots, k, h_{i}$ dans $\mathbb{K}[[X]](M)$. En outre, g appartient à $\mathcal{I}$ si et seulement si $h_{0}=0$.

On en déduit alors une nouvelle preuve de la noethérianité de $\mathbb{K}[[X]](M)$. C'est le corollaire 4.9. On obtient également, comme conséquence, le théorème 0.4 suivant, que l'on retrouve en 4.11 . 
0.4. ThÉORÈme. Soient $f_{1}, \ldots, f_{p}$ des éléments de $\mathbb{K}[[X]](M)$. On note $\mathcal{I}$ l'idéal engendré par ces éléments sur $\mathbb{K}[[X]]$ et $\mathcal{I}_{M}$ l'idéal engendré par ces éléments sur $\mathbb{K}[[X]](M)$. Alors $\mathcal{I}_{M}=\mathcal{I} \cap \mathbb{K}[[X]](M)$.

On notera que ce résultat a déjà été obtenu par l'auteur comme corollaire du théorème d'approximation d'Artin dans $\mathbb{K}[[X]](M)$ (cf. [6]).

On trouvera dans le paragraphe 4 des exemples illustrant ces théorèmes.

1. Une méthode de division. Ce paragraphe est constitué de lemmes techniques qui développent une méthode de division inspirée de [2]. Dans un premier temps, le lecteur pourra se contenter de la proposition 1.6 pour aborder les preuves des principaux résultats développées dans les sections suivantes.

Dans la suite, $\mathcal{A}$, munie de la norme $\|\cdot\|$, désignera une algèbre de Banach commutative unitaire. On a, en particulier, pour $a$ et $b$ dans $\mathcal{A}$, $\|a b\| \leq\|a\|\|b\|$.

On considère alors $\mathcal{A}[[X]]$, l'ensemble des séries formelles, d'indéterminées $X=\left(X_{1}, \ldots, X_{s}\right)$, à coefficients dans $\mathcal{A}$. Soit $\varrho=\left(\varrho_{1}, \ldots, \varrho_{s}\right) \in$ $\left(\mathbb{R}_{+}^{*}\right)^{s}$ un poly-rayon. On pose

$$
\|f\|_{\varrho}^{(M)}=\sum_{j=0}^{\infty} \sum_{J \in \mathbb{N}^{s},|J|=j} \frac{\left\|f_{J}\right\|}{M_{j}} \varrho^{J} .
$$

On note alors

$$
\mathcal{A}[[X]](M, \varrho)=\left\{f \in \mathcal{A}[[X]]:\|f\|_{\varrho}^{(M)}<\infty\right\} .
$$

On vérifie aisément que $\|\cdot\|_{\varrho}^{(M)}$ est une norme sur $\mathcal{A}[[X]](M, \varrho)$ et que l'espace $\mathcal{A}[[X]](M, \varrho)$ muni de cette norme est une algèbre de Banach. On pose

$$
\mathcal{A}[[X]](M)=\bigcup_{\varrho} \mathcal{A}[[X]](M, \varrho) .
$$

1.1. Notations. On note aussi

$$
\begin{aligned}
& (\mathcal{A}[[X]](M, \varrho))^{E_{i}} \\
& \quad=\left\{f \in \mathcal{A}[[X]]: \operatorname{Exp}_{X}\left(f(X) X^{E_{i}}\right) \subset \Delta_{i} \text { et }\|f\|_{\varrho}^{\left(M_{+\left|E_{i}\right|}\right)}<\infty\right\}, \\
& R_{\Delta}(M, \varrho)=\left\{f \in \mathcal{A}[[X]](M, \varrho): f_{B}=0 \text { pour tout } B \in \Delta\right\} .
\end{aligned}
$$

On considère alors l'espace produit

$$
H_{\Delta}(M, \varrho)=\underset{i=1}{\stackrel{p}{\times}}(\mathcal{A}[[X]](M, \varrho))^{E_{i}} \times R_{\Delta}(M, \varrho)
$$

muni de la norme

$$
\left\|\left(g_{1}, \ldots, g_{p} ; h\right)\right\|_{\varrho}^{\Delta(M)}=\sum_{i=1}^{p} \varrho^{E_{i}}\left\|g_{i}\right\|_{\varrho}^{\left(M_{+\left|E_{i}\right|}\right)}+\|h\|_{\varrho}^{(M)} .
$$


On vérifie facilement que l'espace $H_{\Delta}(M, \varrho)$ muni de la norme $\|\cdot\|_{\varrho}^{\Delta(M)}$ est un espace de Banach.

1.2. Lemme. Étant donnés $p$ multi-indices $E_{i}, 1 \leq i \leq p$, on leur associe l'application $\phi_{1}$ définie par

$$
\begin{aligned}
\phi_{1}: H_{\Delta}(M, \varrho) & \rightarrow \mathcal{A}[[X]](M, \varrho), \\
\left(g_{1}, \ldots, g_{p} ; h\right) & \mapsto \phi_{1}\left(g_{1}, \ldots, g_{p} ; h\right)=\sum_{i=1}^{p} g_{i} X^{E_{i}}+h .
\end{aligned}
$$

Pour tout multi-rayon $\varrho, \phi_{1}$ est alors une application linéaire bijective de $H_{\Delta}(M, \varrho)$ sur $\mathcal{A}[[X]](M, \varrho)$ telle que

$$
\left\|\phi_{1}\left(g_{1}, \ldots, g_{p} ; h\right)\right\|_{\varrho}^{(M)}=\left\|\left(g_{1}, \ldots, g_{p} ; h\right)\right\|_{\varrho}^{\Delta(M)} .
$$

Preuve. Les vérifications sont faciles, compte tenu du choix des normes.

1.3. Définitions et remarques. Soit $L$ une forme linéaire sur $\mathbb{R}^{s}$ à coefficients strictement positifs.

(1.3.1) Tout d'abord, on remarque que, pour tout $B \in \mathbb{N}^{s}$, il existe un nombre réel positif $\delta_{B}$ tel que $A \in \mathbb{N}^{s}$ et $L(A)-L(B)>0$ implique $L(A)-L(B) \geq \delta_{B}$.

(1.3.2) On applique alors cette remarque aux multi-indices $E_{i}$. Il existe donc, pour tout $i=1, \ldots, p$, des réels strictement positifs $\delta_{i}$ tels que $L(A)-$ $L\left(E_{i}\right)>0$ implique $L(A)-L\left(E_{i}\right) \geq \delta_{i}$.

(1.3.3) Ensuite, soit $\tau>0$ un réel. On notera $\widetilde{\tau}$ le poly-rayon $(\tau, \ldots, \tau)$ associé de $\mathbb{R}_{+}^{* s}$. Soient $E_{i}, i=1, \ldots, p$, des multi-indices de $\mathbb{N}^{s}$. Dans la suite, on note $u_{i}$ et $v_{i}$ des séries arbitraires de $\mathcal{A}[[X]]\left(M, C^{\left|E_{i}\right|} \widetilde{\tau}\right)$, où $C$ est la constante de $\left(\mathrm{H}_{2}\right)$, de la forme

$$
u_{i}=\sum_{b=\left|E_{i}\right|}^{\infty} \sum_{\substack{B \in \mathbb{N}^{s} \\|B|=b \\ L(B)>L\left(E_{i}\right)}} u_{i B} X^{B}, \quad v_{i}=\sum_{b=\left|E_{i}\right|}^{\infty} \sum_{\substack{B \in \mathbb{N}^{s} \\|B|=b}} v_{i_{B}} X^{B}
$$

On remarque alors que, pour tout $i=1, \ldots, p, u_{i}$ et $v_{i}$ sont telles que, pour tout $A$ de $\operatorname{Exp}_{X}\left(u_{i}\right)$, ou pour tout $A$ de $\operatorname{Exp}_{X}\left(v_{i}\right),|A| \geq\left|E_{i}\right|$.

On construit maintenant, à l'aide de séries $u_{i}$ et $v_{i}, i=1, \ldots, p$, vérifiant l'écriture (1.3.3), une application linéaire $\phi_{2}$ qui sera une perturbation de $\phi_{1}$ en ce sens que $\phi_{1}+\phi_{2}$ sera encore une application linéaire bijective de $H_{\Delta}(M, \varrho)$ sur $\mathcal{A}[[X]](M, \varrho)$.

1.4. Lemme. Soit $0<\eta<1$; on note $r=\left(\tau \eta^{l_{1}}, \ldots, \tau \eta^{l_{s}}\right)$. Soient $E_{i}, u_{i}$ et $v_{i}$, pour $i=1, \ldots, p$, comme dans (1.3.3). L'application linéaire $\phi_{2}$, 


$$
\begin{aligned}
& \phi_{2}: H_{\Delta}(M, r) \rightarrow \mathcal{A}[[X]](M, r), \\
&\left(g_{1}, \ldots, g_{p} ; h\right) \mapsto \phi_{2}\left(g_{1}, \ldots, g_{p} ; h\right)=\sum_{i=1}^{p}\left(u_{i}+v_{i}\right) g_{i},
\end{aligned}
$$

est bien définie et vérifie, pour tout réel $\eta, 0<\eta<1$,

$$
\left\|\phi_{2}\right\| \leq \sup _{i=1, \ldots, p}\left\{\tau^{-\left|E_{i}\right|}\left(\eta^{\delta_{i}}\left\|u_{i}\right\|_{\widetilde{\tau}}^{\left(M_{-\left|E_{i}\right|}\right)}+\eta^{-L\left(E_{i}\right)}\left\|v_{i}\right\|_{\widetilde{\tau}}^{\left(M_{-\left|E_{i}\right|}\right)}\right)\right\} .
$$

Preuve.

1.4.1. Première étape. Pour vérifier que $\sum_{i=1}^{p}\left(u_{i}+v_{i}\right) g_{i}$ appartient bien à $\mathcal{A}[[X]](M, r)$, il suffit donc de s'assurer que $\left\|\sum_{i=1}^{p}\left(u_{i}+v_{i}\right) g_{i}\right\|_{r}^{(M)}$ $<\infty$. Il suffit d'abord de le vérifier pour un terme du type $u_{i} g_{i}$. On a, par définition,

$$
\left\|u_{i} g_{i}\right\|_{r}^{(M)}=\sum_{b=0}^{\infty} \sum_{\substack{B \in \mathbb{N}^{s} \\ b=|B|}} \frac{\left\|\sum_{K+L=B} u_{i_{K}} g_{i_{L}}\right\|}{M_{b}} r^{B} .
$$

On obtient alors, en utilisant, d'une part, le fait qu'on travaille dans des algèbres de Banach, et, d'autre part, la convexité logarithmique de la suite $\left\{M_{n}\right\}_{n \in \mathbb{N}}$,

$$
\left\|u_{i} g_{i}\right\|_{r}^{(M)} \leq \sum_{b=0}^{\infty} \sum_{\substack{B \in \mathbb{N}^{s} \\ b=|B|}} \sum_{K+L=B} \frac{\left\|u_{i}\right\|}{M_{k-\left|E_{i}\right|}} \cdot \frac{\left\|g_{i}\right\|}{M_{l+\left|E_{i}\right|}} r^{K+L} .
$$

Le membre de droite de l'inégalité (1.4.1.2) est bien défini puisque, d'après (1.3.3), $u_{i_{K}}=0$ pour $|K|=k<\left|E_{i}\right|$. De plus, par hypothèse $\left(g_{1}, \ldots, g_{p} ; h\right)$ appartient à $H_{\Delta}(M, r)$; cela implique que, pour tout $i=1, \ldots, p$, on a

$$
\left\|g_{i}\right\|_{r}^{\left(M_{+\left|E_{i}\right|}\right)}<\infty
$$

En outre, par hypothèse, pour tout $i=1, \ldots, p$, on a

$$
u_{i} \in \mathcal{A}[[X]]\left(M, C^{\left|E_{i}\right|} \widetilde{\tau}\right),
$$

ce qui signifie, pour tout $i=1, \ldots, p$,

$$
\sum_{k=0}^{\infty} \sum_{\substack{K \in \mathbb{N}^{s} \\|K|=k}} \frac{\left\|u_{i K}\right\|}{M_{k}} C^{\left|E_{i}\right| k} \widetilde{\tau}^{K}<\infty .
$$

Or, en utilisant $\left|E_{i}\right|$ fois l'hypothèse $\left(\mathrm{H}_{2}\right)$, on obtient, pour tout $i=1, \ldots, p$,

$$
\begin{aligned}
M_{k} & \leq C^{k} M_{k-1} \leq C^{k} C^{k-1} M_{k-2} \leq \ldots \\
& \leq C^{-\left(\left|E_{i}\right|-1\right)\left|E_{i}\right| / 2} C^{\left|E_{i}\right| k} M_{k-\left|E_{i}\right|}
\end{aligned}
$$


Ainsi, en utilisant à nouveau (1.3.3), et en minorant $C^{\left|E_{i}\right| k} / M_{k}$ par $C^{\left(\left|E_{i}\right|-1\right)\left|E_{i}\right| / 2} / M_{k-\left|E_{i}\right|}$ dans l'inégalité (1.4.1.4), on obtient

$$
C^{\left(\left|E_{i}\right|-1\right)\left|E_{i}\right| / 2} \sum_{k=\left|E_{i}\right|}^{\infty} \sum_{\substack{K \in \mathbb{N}^{s} \\|K|=k}} \frac{\left\|u_{i K}\right\|}{M_{k-\left|E_{i}\right|}} \widetilde{\tau}^{K}<\infty
$$

ce qui donne exactement, en remarquant que $r \leq \widetilde{\tau}$,

$$
\left\|u_{i}\right\|_{r}^{\left(M_{-\left|E_{i}\right|}\right)}<\infty .
$$

Dans (1.4.1.2) on reconnaît un produit de séries; on a alors

$$
\begin{aligned}
\left\|u_{i} g_{i}\right\|_{r}^{(M)} & \leq\left(\sum_{b=0}^{\infty} \sum_{\substack{B \in \mathbb{N}^{s} \\
b=|B|}} \frac{\left\|u_{i B}\right\|}{M_{b-\left|E_{i}\right|}} r^{B}\right)\left(\sum_{b=0}^{\infty} \sum_{\substack{B \in \mathbb{N}^{s} \\
|B|=b}} \frac{\left\|g_{i_{B}}\right\|}{M_{b+\left|E_{i}\right|}} r^{B}\right) \\
& \leq\left\|u_{i}\right\|_{r}^{\left(M_{-\left|E_{i}\right|}\right)}\left\|g_{i}\right\|_{r}^{\left(M_{+\left|E_{i}\right|}\right)} .
\end{aligned}
$$

De là, en utilisant (1.4.1.3) et (1.4.1.7), on conclut que, pour tout $i=$ $1, \ldots, p$, on a

$$
\left\|u_{i} g_{i}\right\|_{r}^{(M)}<\infty
$$

et, de la même manière, en utilisant à nouveau (1.3.3),

$$
\begin{gathered}
\left\|v_{i}\right\|_{r}^{\left(M_{-\left|E_{i}\right|}\right)}<\infty, \\
\left\|v_{i} g_{i}\right\|_{r}^{(M)} \leq\left\|v_{i}\right\|_{r}^{\left(M_{-\left|E_{i}\right|}\right)}\left\|g_{i}\right\|_{r}^{\left(M_{+\left|E_{i}\right|}\right)}<\infty .
\end{gathered}
$$

Ceci nous donne bien $\left\|\sum_{i=1}^{p}\left(u_{i}+v_{i}\right) g_{i}\right\|_{r}^{(M)}<\infty$.

1.4.2. Seconde étape. On estime la norme de $\phi_{2}$. On a, par définition,

$$
\left\|\phi_{2}\left(g_{1}, \ldots, g_{p} ; h\right)\right\|_{r}^{(M)}=\left\|\sum_{i=1}^{p}\left(u_{i}+v_{i}\right) g_{i}\right\|_{r}^{(M)} .
$$

Ainsi, en appliquant l'inégalité triangulaire et le fait qu'on se trouve dans des algèbres de Banach, on obtient, d'après (1.4.1.8) et (1.4.1.11),

$$
\begin{aligned}
& \left\|\phi_{2}\left(g_{1}, \ldots, g_{p}, h\right)\right\|_{r}^{(M)} \\
& \leq \sum_{i=1}^{p}\left(\left\|u_{i}\right\|_{r}^{\left(M_{-\left|E_{i}\right|} \mid\right.}\left\|g_{i}\right\|_{r}^{\left(M_{+\left|E_{i}\right|}\right)}+\left\|v_{i}\right\|_{r}^{\left(M_{-\left|E_{i}\right|}\right)}\left\|g_{i}\right\|_{r}^{\left(M_{+\left|E_{i}\right|}\right)}\right) \\
& \leq \sum_{i=1}^{p} r^{E_{i}}\left\|g_{i}\right\|_{r}^{\left(M_{+\left|E_{i}\right|}\right)}\left(\left\|u_{i}\right\|_{r}^{\left(M_{-\left|E_{i}\right|}\right)} r^{-E_{i}}+\left\|v_{i}\right\|_{r}^{\left(M_{-\left|E_{i}\right|}\right)} r^{-E_{i}}\right) .
\end{aligned}
$$


Or, on a, d'après (1.3.2) et la définition de $r$,

$$
\begin{aligned}
\left\|u_{i}\right\|_{r}^{\left(M_{-\left|E_{i}\right|}\right)} r^{-E_{i}} & =\sum_{b=0}^{\infty} \sum_{\substack{B \in \mathbb{N}^{s} \\
(B)>L\left(E_{i}\right) \\
|B|=b}} \frac{\left\|u_{i B}\right\|}{M_{b-\left|E_{i}\right|}} \tau^{b-\left|E_{i}\right|} \eta^{L\left(B-E_{i}\right)} \\
& \leq \tau^{-\left|E_{i}\right|} \eta^{\delta_{i}}\left\|u_{i}\right\|_{\widetilde{\tau}}^{\left(M_{-\left|E_{i}\right|}\right)}
\end{aligned}
$$

De même, on a, puisque $0<\eta<1$,

$$
\begin{aligned}
\left\|v_{i}\right\|_{r}^{\left(M_{-\left|E_{i}\right|}\right)} r^{-E_{i}} & \leq \sum_{b=0}^{\infty} \sum_{\substack{B \in \mathbb{N}^{s} \\
|B|=b}} \frac{\left\|v_{i B}\right\|}{M_{b-\left|E_{i}\right|}} \tau^{b-\left|E_{i}\right|} \eta^{L\left(B-E_{i}\right)} \\
& \leq \tau^{-E_{i}} \eta^{-L\left(E_{i}\right)}\left\|v_{i}\right\|_{\widetilde{\tau}}^{\left(M_{-\left|E_{i}\right|}\right)}
\end{aligned}
$$

On obtient alors le résultat escompté en remarquant

$$
\sum_{i=1}^{p} r^{E_{i}}\left\|g_{i}\right\|_{r}^{\left(M_{+\left|E_{i}\right|}\right)} \leq\left\|\left(g_{1}, \ldots, g_{p}, h\right)\right\|_{r}^{\Delta(M)} .
$$

1.5. Lemme De Perturbation. Si on a

$$
\eta^{\delta_{i}}\left\|u_{i}\right\|_{\widetilde{\tau}}^{\left(M_{-\left|E_{i}\right|}\right)}<\tau^{\left|E_{i}\right|} / 2
$$

et

$$
\left\|v_{i}\right\|_{\widetilde{\tau}}^{\left(M_{-\left|E_{i}\right|}\right)}<\frac{1}{2} \tau^{\left|E_{i}\right|} \eta^{L\left(E_{i}\right)},
$$

alors l'application $\phi_{1}+\phi_{2}$ est un isomorphisme entre les deux espaces de Banach $H_{\Delta}(M, r)$ et $\mathcal{A}[[X]](M, r)$.

Preuve. Pour tout $\varrho \in \mathbb{R}_{+}^{* s}$, on a donc trouvé, d'après le lemme 1.2, une isométrie bijective $\phi_{1}$ entre les deux espaces de Banach $H_{\Delta}(M, \varrho)$ et $\mathcal{A}[[X]](M, \varrho)$. On peut donc écrire

$$
\phi_{1}+\phi_{2}=\phi_{1}\left(\mathrm{Id}+\phi_{1}^{-1} \phi_{2}\right) .
$$

Ainsi, si $\left\|\phi_{2}\right\|<1$, l'application $\phi_{1}+\phi_{2}$ sera inversible. Pour tout $\eta, 0<$ $\eta<1$, on note $r=\left(\tau \eta^{l_{1}}, \ldots, \tau \eta^{l_{s}}\right)$. Si on a (1.5.1) et (1.5.2), alors, d'après la proposition 1.4 , on conclut $\left\|\phi_{2}\right\|<1$.

On obtient alors, comme conséquence des lemmes 1.2, 1.4 et 1.5, un théorème de division dans les séries formelles à coefficients dans des algèbres de Banach commutatives et unitaires.

1.6. Proposition (de $L$-division dans $\mathcal{A}[[X]](M)$ ). Soient $\mathcal{A}$ une algèbre de Banach commutative unitaire et $\tau_{1}>0$. Soient $f_{1}, \ldots, f_{p}$ des éléments 
de $\mathcal{A}[[X]]\left(M, \widetilde{\tau_{1}}\right)$ et soient $E_{1}, \ldots, E_{p}$ des multi-indices de $\mathbb{N}^{s}$ tels que, pour tout $i=1, \ldots, p$, on ait

(i) $\left(f_{i}\right)_{B}=0$ pour tout $B \in \mathbb{N}^{s},|B|<\left|E_{i}\right|$,

(ii) $\left(f_{i}\right)_{E_{i}}=1+a_{i}$,

avec 1 l'élément unité de l'algèbre $\mathcal{A}$ et $a_{i}$ un élément de $\mathcal{A}$. Soit $L$ une forme linéaire à coefficients strictement positifs de $\mathbb{R}^{s}$. Alors on écrit, pour tout $i=1, \ldots, p$,

$$
f_{i}(X)=X^{E_{i}}+u_{i}(X)+v_{i}(X)
$$

avec

$$
\begin{aligned}
& u_{i}(X)=\sum_{j=\left|E_{i}\right|}^{\infty} \sum_{\substack{|J|=j \\
L(J)>L\left(E_{i}\right)}}\left(f_{i}\right)_{J} X^{J}, \\
& v_{i}(X)=\sum_{j=\left|E_{i}\right|}^{\infty} \sum_{\substack{|J|=j \\
(J) \leq L\left(E_{i}\right) \\
J \neq E_{i}}}\left(f_{i}\right)_{J} X^{J}+a_{i} X^{E_{i}} .
\end{aligned}
$$

On a les conclusions suivantes.

(1) Il existe $\tau_{2}, 0<\tau_{2} \leq \tau_{1}$, tel que, pour tout $0<\tau \leq \tau_{2}$, il existe $\eta(\tau)$, $0<\eta(\tau)<1$, tel que, pour tout $i=1, \ldots, p$, on a

$$
\eta(\tau)^{\delta_{i}}\left\|u_{i}\right\|_{\tilde{\tau}}^{\left(M_{-\left|E_{i}\right|}\right)}<\tau^{\left|E_{i}\right|} / 2 .
$$

(2) Si, de plus, on a

$$
\left\|v_{i}\right\|_{\widetilde{\tau}}^{\left(M_{-\left|E_{i}\right|}\right)}<\frac{1}{2} \tau^{\left|E_{i}\right|} \eta(\tau)^{L\left(E_{i}\right)},
$$

alors, il existe un poly-rayon $\mu$ de $\mathbb{R}_{+}^{* s}$ strictement inférieur à $\widetilde{\tau}$, tel que, pour tout élément $g$ de $\mathcal{A}[[X]](M, \widetilde{\tau})$, il existe des séries $g_{1}, \ldots, g_{p}, h$ de $\mathcal{A}[[X]](M, \mu)$ telles que l'on ait

$$
g=\sum_{i=1}^{p} f_{i} g_{i}+h
$$

avec

$$
\begin{gathered}
\operatorname{Exp}_{X}\left(g_{i}(X) X^{E_{i}}\right) \subset \Delta_{i}, \quad i=1, \ldots, p, \\
h=\sum_{b=0}^{\infty} \sum_{\substack{B \in \mathbb{N}^{s} \\
|B|=b}} h_{B} X^{B} \quad \text { avec } h_{B}=0 \text { pour } B \in \Delta,
\end{gathered}
$$

où $\mathbb{N}^{s}=\Delta_{1} \cup \ldots \cup \Delta_{p} \cup\left(\mathbb{N}^{s}-\Delta\right)$ est la partition de $\mathbb{N}^{s}$ associée aux $E_{i}, 1 \leq i \leq p$. De plus, $\left(g_{1}, \ldots, g_{p} ; h\right)$ est donné par un opérateur linéaire continu. 
Preuve. Par hypothèse, pour tout $i=1, \ldots, p, f_{i}$ appartient à $\mathcal{A}[[X]]\left(M, \widetilde{\tau_{1}}\right)$, et donc en utilisant alors l'hypothèse $\left(\mathrm{H}_{2}\right)$ sur la suite $\left\{M_{n}\right\}_{n \in \mathbb{N}}$, et en procédant comme dans (1.4.1.5), on obtient, pour tout $i=1, \ldots, p$,

$$
\left\|f_{i}\right\|_{\widetilde{\tau}_{1} / C^{\left|E_{i}\right|}}^{\left(M_{-\left|E_{i}\right|}\right)}<\infty
$$

On note $e=\min _{i=1, \ldots, p}\left(1 / C^{\left|E_{i}\right|}\right)$. En posant alors $\tau_{2}=e \tau_{1}$, on obtient, pour tout $i=1, \ldots, p$,

$$
\left\|u_{i}\right\|_{\widetilde{\tau}_{2}}^{\left(M_{-\left|E_{i}\right|}\right)}<\infty \quad \text { et }\left\|v_{i}\right\|_{\widetilde{\tau}_{2}}^{\left(M_{-\left|E_{i}\right|}\right)}<\infty .
$$

Ainsi, pour tout $\tau \leq \tau_{2}$, on a

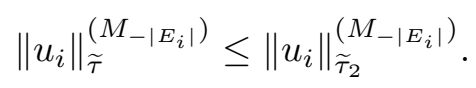

Pour tout $\tau \leq \tau_{2}$, on peut choisir $\eta(\tau)<1$, assez petit, pour que l'on ait

$$
\eta(\tau)^{\delta_{i}}\left\|u_{i}\right\|_{\widetilde{\tau}_{2}}^{\left(M_{-\left|E_{i}\right|}\right)}<\tau^{\left|E_{i}\right|} / 2
$$

Cela donne, en utilisant (1.6.6),

$$
\eta(\tau)^{\delta_{i}}\left\|u_{i}\right\|_{\tilde{\tau}^{\left(M_{-\left|E_{i}\right|}\right)}}^{\left(\tau^{\left|E_{i}\right|} / 2\right.}
$$

On obtient donc le (1) de la proposition.

De plus, pour avoir le (2) de la proposition, il suffit de remarquer d'abord que (1.6.8) est exactement la condition (1.5.1). Si on a, dans le même temps,

$$
\left\|v_{i}\right\|_{\tilde{\tau}}^{\left(M_{-\left|E_{i}\right|}\right)}<\frac{1}{2} \tau^{\left|E_{i}\right|} \eta(\tau)^{L\left(E_{i}\right)}
$$

la condition (1.5.2) est réalisée. On se trouve alors sous les hypothèses du lemme 1.5 et l'application

$$
\begin{aligned}
\phi_{1}+\phi_{2}: H_{\Delta}(M, r) & \rightarrow \mathcal{A}[[X]](M, r), \\
\left(g_{1}, \ldots, g_{p} ; h\right) \mapsto\left(\phi_{1}+\phi_{2}\right)\left(g_{1}, \ldots, g_{p} ; h\right) & =\sum_{i=1}^{p} g_{i} Y^{E_{i}}+h+\sum_{i=1}^{p}\left(u_{i}+v_{i}\right) g_{i},
\end{aligned}
$$

avec $r=\left(\tau \eta^{l_{1}}, \ldots, \tau \eta^{l_{s}}\right)$, est une application linéaire bijective. Alors, pour tout élément $g$ de $\mathcal{A}[[X]](M, \widetilde{\tau})$, il existe un unique $p+1$-uplet

$$
\left(g_{1}, \ldots, g_{p} ; h\right) \in H_{\Delta}(M, r)
$$

tel que

$$
\begin{aligned}
& g=\sum_{i=1}^{p}\left(X^{E_{i}}+u_{i}+v_{i}\right) g_{i}+h, \\
& h=\sum_{b=0}^{\infty} \sum_{\substack{B \in \mathbb{N}^{s} \\
|B|=b}} h_{B} X^{B} \quad \text { avec } h_{B}=0 \text { pour } B \in \Delta .
\end{aligned}
$$


En remarquant que, pour tout $i=1, \ldots, p, X^{E_{i}}+u_{i}+v_{i}=f_{i}$, on obtient exactement $g=\sum_{i=1}^{p} f_{i} g_{i}+h$. On a alors, pour tout $i=1, \ldots, p$, $\left\|g_{i}\right\|_{r}^{\left(M_{+\left|E_{i}\right|}\right)}<\infty$ et $\|h\|_{r}^{(M)}<\infty$. En utilisant l'estimation de (1.4.1.5), on peut affirmer que, pour tout $i=1, \ldots, p$,

$$
\left\|g_{i}\right\|_{r / C^{\left|E_{i}\right|}}^{(M)}<\infty
$$

ce qui donne le résultat avec $\mu=r e$.

Remarque. Si pour tout $i=1, \ldots, p, v_{i}$ est identiquement nul, la condition (1.5.2) disparaît. La condition (1.5.1) est toujours réalisable. La division a lieu.

\section{Division dans l'anneau des séries formelles à croissance con-} trôlée. On peut maintenant appliquer la proposition 1.6 pour obtenir des théorèmes de division avec estimations dans l'anneau des séries formelles à croissance contrôlée.

2.1. Définitions et notations. Soient $f_{1}, \ldots, f_{p}$ des éléments de $\mathbb{K}[[Y]][[X]]$. Soient $L$ une forme linéaire à coefficients strictement positifs de $\mathbb{R}^{s}$ et $E_{1}, \ldots, E_{p}$ des multi-indices de $\mathbb{N}^{s}$. On dira que la famille $f_{i}$, $1 \leq i \leq p$, est $\left(E_{1}, \ldots, E_{p} ; L\right)$-régulière dans $\mathbb{K}[[Y]][[X]]$ si les conditions suivantes sont vérifiées :

(i) $\left(f_{i}\right)_{B}(Y)=0$ pour tout $B \in \mathbb{N}^{s},|B|<\left|E_{i}\right|$,

(ii) $\left(f_{i}\right)_{E_{i}}(0) \neq 0$,

(iii) $\left(f_{i}\right)_{B}(0)=0$ pour tout $B \in \mathbb{N}^{s}, B \neq E_{i}$ et $L(B) \leq L\left(E_{i}\right)$.

Il est facile de vérifier que, si on se donne des séries formelles $f_{i}, 1 \leq i \leq p$, de $q+s$ variables, on peut toujours trouver une partition $X, Y$ sur l'ensemble des variables, des multi-indices $E_{1}, \ldots, E_{p}$ et une forme linéaire $L$ tels que la famille $\left(f_{1}, \ldots, f_{p}\right)$ soit $\left(E_{1}, \ldots, E_{p} ; L\right)$-régulière dans $\mathbb{K}[[Y]][[X]]$. On donne un exemple. On considère $f_{1}\left(X_{1}, X_{2}, X_{3}\right)=X_{1}^{2} X_{2}^{2}+X_{1} X_{2}^{3}+X_{1}^{3} X_{2} X_{3}+X_{1}^{7}$ et $f_{2}\left(X_{1}, X_{2}, X_{3}\right)=X_{1}^{2} X_{2}+X_{1}^{3} X_{3}+X_{1}^{2} X_{2}^{2}+X_{1}^{4}$. On pose $E_{1}=(2,2)$ et $E_{2}=(2,1)$. Soit $L$ la forme linéaire de $\mathbb{R}^{2}$ telle que $L\left(i_{1}, i_{2}\right)=i_{1}+2 i_{2}$. On vérifie facilement que la famille $\left(f_{1}, f_{2}\right)$ est $\left(E_{1}, E_{2} ; L\right)$-régulière dans $\mathbb{K}\left[\left[X_{3}\right]\right]\left[\left[X_{1}, X_{2}\right]\right]$. En revanche, elle ne sera jamais régulière dans $\mathbb{K}\left[\left[X_{2}, X_{3}\right]\right]\left[\left[X_{1}\right]\right]$.

Soit $\left\{N_{n}\right\}_{n \in \mathbb{N}}$ une suite de réels positifs vérifiant les conditions $\left(\mathrm{H}_{1}\right)$ et $\left(\mathrm{H}_{2}\right)$. Soit $\mathbb{K}=\mathbb{R}$ ou $\mathbb{C}$. Dans la suite, on prendra pour $\mathcal{A}$ l'algèbre de Banach des séries formelles $\mathbb{K}[[Y]](N, \alpha)$, avec $Y=\left(Y_{1}, \ldots, Y_{q}\right)$, munie de la norme $\|\cdot\|_{\alpha}^{(N)}$, si $\alpha=\left(\alpha_{1}, \ldots, \alpha_{q}\right)$ est un poly-rayon de $\mathbb{R}_{+}^{* q}$. On a donc, en suivant les notations du paragraphe 1.1 , pour tout $f \in \mathcal{A}[[X]]$, 


$$
\|f\|_{(N, \alpha)(\varrho)}^{(M)}=\sum_{j=0}^{\infty} \sum_{J \in \mathbb{N}^{s},|J|=j} \frac{\left\|f_{J}\right\|_{\alpha}^{(N)}}{M_{j}} \varrho^{J}
$$

et

$$
(\mathbb{K}[[Y]](N, \alpha))[[X]](M, \varrho)=\left\{f \in\left(\mathbb{K}[[Y]](N, \alpha)[[X]]:\|f\|_{(N, \alpha)(\varrho)}^{(M)}<\infty\right\} .\right.
$$

L'espace $(\mathbb{K}[[Y]](N, \alpha))[[X]](M, \varrho)$ muni de la norme $\|\cdot\|_{(N, \alpha)(\varrho)}^{(M)}$ est une algèbre de Banach.

2.2. ThÉORÈme (de $L$-division). Soient $f_{1}, \ldots, f_{p}$ des éléments de $\left(\mathbb{K}[[Y]]\left(N, \widetilde{\nu}_{1}\right)\right)[[X]]\left(M, \widetilde{\tau}_{1}\right)$. Soient $L$ une forme linéaire à coefficients strictement positifs de $\mathbb{R}^{s}$ et $E_{1}, \ldots, E_{p}$ des multi-indices de $\mathbb{N}^{s}$ tels que la famille $\left(f_{1}, \ldots, f_{p}\right)$ soit $\left(E_{1}, \ldots, E_{p} ; L\right)$-régulière dans $\mathbb{K}[[Y]][[X]]$. Alors il existe $\tau_{2}, 0<\tau_{2} \leq \tau_{1}$, tel que, pour tout $0<\tau \leq \tau_{2}$ et pour tout $\nu \leq \nu_{1}$, il existe un poly-rayon $\mu$ de $\mathbb{R}_{+}^{* s}, \mu<\widetilde{\tau}$, tel que, pour tout élément $g$ de $(\mathbb{K}[[Y]](N, \widetilde{\nu}))[[X]](M, \widetilde{\tau})$, il existe des uniques séries $g_{1}, \ldots, g_{p}, h$ de $(\mathbb{K}[[Y]](N, \widetilde{\nu}))[[X]](M, \mu)$ telles que l'on ait

$$
\begin{gathered}
g=\sum_{i=1}^{p} f_{i} g_{i}+h, \\
\operatorname{Exp}_{X}\left(g_{i}(Y, X) X^{E_{i}}\right) \subset \Delta_{i}, \quad i=1, \ldots, p, \\
h=\sum_{b=0}^{\infty} \sum_{\substack{B \in \mathbb{N}^{s} \\
|B|=b}} h_{B} X^{B} \quad \text { avec } h_{B}=0 \text { pour } B \in \Delta,
\end{gathered}
$$

où $\mathbb{N}^{s}=\Delta_{1} \cup \ldots \cup \Delta_{p} \cup\left(\mathbb{N}^{s}-\Delta\right)$ est la partition de $\mathbb{N}^{s}$ associée aux $E_{i}, 1 \leq i \leq p$. De plus, $\left(g_{1}, \ldots, g_{p} ; h\right)$ est donné par un opérateur linéaire continu.

Preuve. On reprend la démarche de la preuve de la proposition 1.6. On peut évidemment supposer $\left(f_{i}\right)_{E_{i}}(0)=1$. On a alors $\left(f_{i}\right)_{E_{i}}(Y)=1+a_{i}(Y)$ avec $a_{i}(0)=0$. On écrit donc, d'après les conditions (i)-(iii),

$$
f_{i}(Y, X)-X^{E_{i}}=u_{i}(Y, X)+v_{i}(Y, X)
$$

avec

$$
u_{i}(Y, X)=\sum_{b=\left|E_{i}\right|}^{\infty} \sum_{\substack{B \in \mathbb{N}^{s} \\ L(B)>L\left(E_{i}\right) \\|B|=b}}\left(f_{i}\right)_{B}(Y) X^{B}
$$

$$
v_{i}(Y, X)=\sum_{b=\left|E_{i}\right|}^{\infty} \sum_{\substack{\left.B \in \mathbb{N}^{s} \\ B \neq E_{i} \\ L\right) \leq L\left(E_{i}\right) \\|B|=b}}\left(f_{i}\right)_{B}(Y) X^{B}+a_{i}(Y) X^{E_{i}}, \quad v_{i}(0, X)=0 .
$$


D'autre part, $f_{i}$ appartient à $\left(\mathbb{K}[[Y]]\left(N, \widetilde{\nu_{1}}\right)\right)[[X]]\left(M, \widetilde{\tau_{1}}\right)$ pour tout $i=$ $1, \ldots, p$. En utilisant alors l'hypothèse $\left(\mathrm{H}_{2}\right)$ sur la suite $\left\{M_{n}\right\}_{n \in \mathbb{N}}$, et en procédant comme dans (1.4.1.5), on obtient, pour tout $i=1, \ldots, p$,

$$
\left\|f_{i}\right\|_{\left(N, \widetilde{\nu}_{1}\right)\left(\widetilde{\tau}_{1} / C^{\left|E_{i}\right|}\right)}^{\left(M_{-}\right)}<\infty .
$$

En notant toujours $e=\min _{i=1, \ldots, p}\left(1 / C^{\left|E_{i}\right|}\right)$, on pose $\tau_{2}=e \tau_{1}$. On a donc, pour tout $i=1, \ldots, p$,

$$
\left\|u_{i}\right\|_{\left(N, \widetilde{\nu}_{1}\right)\left(\widetilde{\tau}_{2}\right)}^{\left(M_{-\mid E_{i}}\right)}<\infty \quad \text { et }\left\|v_{i}\right\|_{\left(N, \widetilde{\nu}_{1}\right)\left(\widetilde{\tau}_{2}\right)}^{\left(M_{-}\right)}<\infty .
$$

Et donc, pour tout $\nu \leq \nu_{1}$ et pour tout $\tau \leq \tau_{2}$, on a

$$
\left\|u_{i}\right\|_{(N, \widetilde{\nu})(\widetilde{\tau})}^{\left(M_{-} \mid E_{i}\right)}=\sum_{b=\left|E_{i}\right|}^{\infty} \sum_{\substack{B \in \mathbb{N}^{s} \\ L(B)>L\left(E_{i}\right) \\|B|=b}} \frac{\left\|\left(f_{i}\right)_{B}\right\|_{\widetilde{\nu}}^{(N)}}{M_{b-\left|E_{i}\right|}} \tau^{b} \leq\left\|u_{i}\right\|_{\left(N, \widetilde{\nu}_{1}\right)\left(\tilde{\tau}_{2}\right)}^{\left(M_{-}\right)}
$$

On a aussi, pour tout coefficient $\left(f_{i}\right)_{B}$ tel que $\left(f_{i}\right)_{B}(0)=0$,

$$
\begin{aligned}
\left\|\left(f_{i}\right)_{B}\right\|_{\widetilde{\nu}}^{(N)} & =\sum_{a=1}^{\infty} \sum_{\substack{A \in \mathbb{N}^{q} \\
|A|=a}} \frac{\left\|\left(f_{i}\right)_{A, B}\right\|}{N_{a}} \nu^{a}=\nu \sum_{a=1}^{\infty} \sum_{\substack{A \in \mathbb{N}^{q} \\
|A|=a}} \frac{\left\|\left(f_{i}\right)_{A, B}\right\|}{N_{a}} \nu^{a-1} \\
& \leq \nu \sum_{a=1}^{\infty} \sum_{\substack{A \in \mathbb{N}^{q} \\
|A|=a}} \frac{\left\|\left(f_{i}\right)_{A, B}\right\|}{N_{a}} \nu_{1}^{a-1} \leq \frac{\nu}{\nu_{1}}\left\|\left(f_{i}\right)_{B}\right\|_{\widetilde{\nu}_{1}}^{(N)}
\end{aligned}
$$

Et donc, pour tout $\nu \leq \nu_{1}$ et pour tout $\tau \leq \tau_{2}$, on a

$$
\begin{aligned}
\left\|v_{i}\right\|_{(N, \widetilde{\nu})(\widetilde{\tau})}^{\left(M_{-\left|E_{i}\right|}\right)} & =\sum_{b=\left|E_{i}\right|}^{\infty} \sum_{\substack{B \in \mathbb{N}^{s} \\
L(B) \leq L\left(E_{i}\right) \\
|B|=b}} \frac{\left\|\left(f_{i}\right)_{B}\right\|_{\widetilde{\nu}}^{(N)}}{M_{b-\left|E_{i}\right|}} \tau^{b} \\
& \leq \sum_{b=\left|E_{i}\right|}^{\infty} \sum_{\substack{B \in \mathbb{N}^{s} \\
L(B) \leq L\left(E_{i}\right) \\
|B|=b}} \frac{\nu}{\nu_{1}} \cdot \frac{\left\|\left(f_{i}\right)_{B}\right\|_{\widetilde{\nu}_{1}}^{(N)}}{M_{b-\left|E_{i}\right|}}\left(\tau_{2}\right)^{b} \\
& \leq \frac{\nu}{\nu_{1}}\left\|v_{i}\right\|_{\left(N, \mathbb{\nu}_{1}\right)\left(\tilde{\tau}_{2}\right)}^{\left(M_{2} \mid\right)} .
\end{aligned}
$$

Pour tout $\tau \leq \tau_{2}$, on choisit $\eta(\tau)<1$, assez petit pour que l'on ait

$$
\eta(\tau)^{\delta_{i}}\left\|u_{i}\right\|_{\left(N, \widetilde{\nu}_{1}\right)\left(\widetilde{\tau}_{2}\right)}^{\left(M_{-}\right)}<\tau^{\left|E_{i}\right|} / 2 .
$$

Cela donne, en utilisant (2.2.7),

$$
\eta(\tau)^{\delta_{i}}<\frac{1}{2} \tau^{\left|E_{i}\right|}\left(\left\|u_{i}\right\|_{(N, \widetilde{\nu})(\widetilde{\tau})}^{\left(M_{-} \mid E_{i}\right)}\right)^{-1} \quad \text { pour tout } \nu \leq \nu_{1} .
$$


On choisit ensuite $\nu=\nu(\tau, \eta(\tau))$ inférieur à $\nu_{1}$, assez petit pour que l'on ait

$$
\frac{\nu}{\nu_{1}}\left\|v_{i}\right\|_{\left(N, \widetilde{\nu}_{1}\right)\left(\widetilde{\tau}_{2}\right)}^{\left(M_{-} \mid E_{1}\right)}<\frac{1}{2} \tau^{\left|E_{i}\right|} \eta(\tau)^{L\left(E_{i}\right)} .
$$

Cela donne, en utilisant (2.2.9),

$$
\left\|v_{i}\right\|_{(N, \widetilde{\nu})(\widetilde{\tau})}^{\left(M_{-} \mid E_{1}\right)}<\frac{1}{2} \tau^{\left|E_{i}\right|} \eta(\tau)^{L\left(E_{i}\right)} .
$$

Les conditions (1) et (2) de la proposition générale de $L$-division 1.6 sont données respectivement par (2.2.11) et (2.2.13). Il existe donc un poly-rayon $\mu<\widetilde{\tau}$ tel que les conclusions de cette proposition soient vérifiées. De plus, si $\left(g_{1}, \ldots, g_{p} ; h\right)$ et $\left(g_{1}^{\prime}, \ldots, g_{p}^{\prime} ; h^{\prime}\right)$ sont deux $p+1$-uplets vérifiant $(2.2 .1)-$ (2.2.3), alors on a, pour tout $i=1, \ldots, p, g_{i}^{\prime}=g_{i}$ et $h^{\prime}=h$. Il suffit de se référer à la version correspondante du théorème de division formelle dans [1].

2.3. Remarques. (a) Dans le cas où $M_{n}=1$, pour tout $n \in \mathbb{N}$, l'hypothèse (i) du théorème 2.2 est superflue. Le lecteur s'en convaincra en reprennant la démonstration de la proposition 1.4 et du théorème 2.2. On notera l'analogie avec la preuve du théorème de Briançon [2].

(b) On déduit aisément du théorème 2.2 un théorème de division dans $(\mathbb{K}[[Y]](N))[[X]](M)$. Dans la suite, si $q=0$, c'est-à-dire si les séries formelles sont à coefficients dans $\mathbb{K}$, on dira du théorème 2.2 qu'il est sans paramètres. On a alors l'énoncé suivant, annoncé en 0.2 .

2.4. ThÉorème. Soient $f_{1}, \ldots, f_{p}$ dans $\mathbb{K}[[X]](M)$. Soient $L$ sur $\mathbb{R}^{s}$ une forme linéaire à coefficients strictement positifs et $E_{1}, \ldots, E_{p}$ des multiindices de $\mathbb{N}^{s}$ tels que la famille $\left(f_{1}, \ldots, f_{p}\right)$ soit $\left(E_{1}, \ldots, E_{p} ; L\right)$-régulière dans $\mathbb{K}[[X]]$. Alors, pour tout élément $g$ de $\mathbb{K}[[X]](M)$, il existe des uniques séries $g_{1}, \ldots, g_{p}, h$ de $\mathbb{K}[[X]](M)$ telles que l'on ait

$$
\begin{gathered}
g=\sum_{i=1}^{p} f_{i} g_{i}+h, \\
\operatorname{Exp}_{X}\left(g_{i}(X) X^{E_{i}}\right) \subset \Delta_{i}, \quad i=1, \ldots, p, \\
h=\sum_{b=0}^{\infty} \sum_{\substack{B \in \mathbb{N}^{s} \\
|B|=b}} h_{B} X^{B} \quad \text { avec } h_{B}=0 \text { pour } B \in \Delta,
\end{gathered}
$$

où $\mathbb{N}^{s}=\Delta_{1} \cup \ldots \cup \Delta_{p} \cup\left(\mathbb{N}^{s}-\Delta\right)$ est la partition de $\mathbb{N}^{s}$ associée aux $E_{i}, 1 \leq i \leq p$. De plus, $\left(g_{1}, \ldots, g_{p} ; h\right)$ est donné par un opérateur linéaire continu.

De plus, cette division est toujours possible. Il est facile de vérifier qu'il existe toujours une forme linéaire $L$ et des multi-indices $E_{1}, \ldots, E_{p}$ tels que $\left(f_{1}, \ldots, f_{p}\right)$ soit $\left(E_{1}, \ldots, E_{p} ; L\right)$-régulière. 


\section{APPLICATIONS}

3. La préparation et la division de Weierstrass. On retrouve, comme conséquence du théorème 2.4, une version des théorèmes de division et de préparation de Weierstrass dans $\mathbb{K}[[X]](M)$ (cf. [3]). Pour cela, on rappelle une notion de $p$-régularité introduite par J. Chaumat et A.-M. Chollet [3].

Soit $f$ un élément de $\mathbb{K}[[X]]$,

$$
f(X)=\sum_{j=0}^{\infty} \sum_{\substack{J \in \mathbb{N}^{s} \\|J|=j}} f_{J} X^{J}
$$

et $p$ un entier. On dira que $f$ est $p$-régulière par rapport à la variable $X_{s}$ si

$f_{J}=0$ pour tout muti-indice $J$ tel que $|J|<p$ et $f_{(0, \ldots, 0, p)} \neq 0$.

Ainsi, pour tout élément $f$ de $\mathbb{K}[[X]]$ non identiquement nul, il existe $p$ tel que, après un éventuel changement de variable linéaire, $f$ soit $p$-régulière par rapport à la variable $X_{s}$. On note

$$
\operatorname{ord}(f)=\min \left(j: f_{J} \neq 0\right) .
$$

3.1. ThÉorème (Division de Weierstrass [3]). Soit $f \in \mathbb{K}[[X]](M)$ une série formelle, p-régulière par rapport à la variable $X_{s}$. Alors, pour tout $g \in \mathbb{K}[[X]](M)$, on peut écrire

$$
g\left(X_{1}, \ldots, X_{s}\right)=q\left(X_{1}, \ldots, X_{s}\right) f\left(X_{1}, \ldots, X_{s}\right)+\sum_{k=0}^{p-1} r_{k}\left(X_{1}, \ldots, X_{s-1}\right) X_{s}^{k}
$$

avec $q \in \mathbb{K}[[X]](M), r_{k} \in \mathbb{K}[[X]](M), k=0, \ldots, p-1$, uniques.

Cette proposition n'est autre que le théorème 2.4 (voir remarque 2.3(b)). En effet, on considère une forme linéaire $L$ à coefficients strictement positifs et indépendants sur $\mathbb{Z}$ telle que $L(0, \ldots, 0, p)<L(J)$ pour tout multi-indice $J, J \neq(0, \ldots, 0, p)$, tel que $f_{J} \neq 0$. La série $f$ est alors $((0, \ldots, 0, p) ; L)$ régulière dans $\mathbb{K}[[X]]$.

3.2. ThÉorème (Préparation de Weierstrass $[3])$. Soit $f \in \mathbb{K}[[X]](M)$ une série formelle, $p$-régulière par rapport à la variable $X_{s}$. Il existe alors des uniques séries formelles $U \in \mathbb{K}[[X]](M)$ inversible et $r_{0}, \ldots, r_{p-1} \in$ $\mathbb{K}\left[\left[X_{1}, \ldots, X_{s-1}\right]\right](M)$ telles que

$$
f\left(X_{1}, \ldots, X_{s}\right)=U\left(X_{1}, \ldots, X_{s}\right)\left(X_{s}^{p}-\sum_{k=0}^{p-1} r_{k}\left(X_{1}, \ldots, X_{s-1}\right) X_{s}^{k}\right) .
$$

En outre, on a, pour tout $k=0, \ldots, p-1, \operatorname{ord}\left(r_{k}\left(X_{1}, \ldots, X_{s-1}\right)\right) \geq p-k$. 
3.3. Proposition. Soit $f \in \mathbb{K}\left[\left[X_{1}, \ldots, X_{s-1}\right]\right](M)\left[X_{s}\right]$ une série formelle polynômiale et régulière d'ordre $p$ en $X_{s}$. Alors, pour tout $g \in$ $\mathbb{K}\left[\left[X_{1}, \ldots, X_{s-1}\right]\right](M)\left[X_{s}\right]$, on peut écrire

$$
g\left(X_{1}, \ldots, X_{s}\right)=q\left(X_{1}, \ldots, X_{s}\right) f\left(X_{1}, \ldots, X_{s}\right)+\sum_{k=0}^{p-1} r_{k}\left(X_{1}, \ldots, X_{s-1}\right) X_{s}^{k}
$$

avec $q \in \mathbb{K}[[X]](M), r_{k} \in \mathbb{K}[[X]](M), k=0, \ldots, p-1$, uniques.

Preuve. D'après la remarque 2.3(a), si la série $f(X)$ appartient à $\mathbb{K}\left[\left[X_{1}, \ldots, X_{s-1}\right]\right](M)\left[X_{s}\right]$, l'anneau des polynômes en $X_{s}$ à coefficients dans $\mathbb{K}\left[\left[X_{1}, \ldots, X_{s-1}\right]\right](M)$, l'hypothèse de $p$-régularité dans les théorèmes 3.1 et 3.2 est superflue. Il suffit d'avoir la régularité d'ordre $p$ de $f$, c'est-à-dire $f\left(0, \ldots, 0, X_{s}\right)=X_{s}^{p} c\left(X_{s}\right)$, avec $c(0) \neq 0$.

3.4. Corollaire. L'anneau $\mathbb{K}[[X]](M)$ est un anneau hensélien.

Preuve. C'est une application directe de la proposition 3.3, en suivant une démonstration classique [5].

3.5. Un exemple. Pour illustrer le théorème 2.4 et plus particulièrement l'importance du choix de la variable par rapport à laquelle on va effectuer la division, on donne un exemple.

Soit $M_{n}=n$ !. Considérons la série formelle $g(X, Y)=\sum_{n=0}^{\infty} M_{2 n} X^{n} Y^{n}$, que nous allons diviser par $f(X, Y)=X+Y^{2}$. Si on effectue cette division par rapport à $X$, les hypothèses du théorème 2.4 sont vérifiées, $\Delta=(1,0)+$ $\mathbb{N}^{2}$ et on obtient

$$
g(X, Y)=f(X, Y) q_{1}(X, Y)+r_{1}(X, Y)
$$

avec

$$
\begin{aligned}
& q_{1}(X, Y)=\sum_{k=1}^{\infty} \sum_{n=0}^{\infty}(-1)^{k-1} M_{2(n+k)} X^{n} Y^{n+3 k-2} \\
& r_{1}(X, Y)=\sum_{n=0}^{\infty}(-1)^{n} M_{2 n} Y^{3 n} .
\end{aligned}
$$

Il est aisé de vérifier que $g, q_{1}$ et $r_{1}$ appartiennent à $\mathbb{K}[[X, Y]](M)$.

En revanche, si on effectue cette division par rapport à $Y^{2}$, on a $\Delta=$ $(0,2)+\mathbb{N}^{2}$ et on obtient

$$
g(X, Y)=f(X, Y) q_{2}(X, Y)+r_{2}(X, Y)
$$

avec

$$
q_{2}(X, Y)=\sum_{k=1}^{\infty} \sum_{n=0}^{\infty}(-1)^{k-1} M_{2 n+4 k} X^{n+3 k-1} Y^{n}
$$




$$
r_{2}(X, Y)=\sum_{n=0}^{\infty}(-1)^{n}\left(M_{4 n} X^{3 n}+M_{4 n+2} X^{3 n+1} Y\right) .
$$

Il est aisé de vérifier que $q_{2}$ et $r_{2}$ appartiennent à $\mathbb{K}[[X, Y]]\left(M^{(2)}\right)$ mais n'appartiennent pas à $\mathbb{K}[[X, Y]](M)$. Le choix de la variable, par rapport à laquelle on effectue la division, est donc fondamental.

4. Division par un idéal. Dans toute la suite, on se place dans l'anneau des séries formelles à coefficients dans $\mathbb{K}$ et on suppose que

(**) $\quad L$ est une forme linéaire dont les coefficients sont strictement positifs et indépendants sur $\mathbb{Z}$.

4.1. Notations. Soient $A$ et $B$ deux multi-indices de $\mathbb{N}^{s}$. On dira que $A$ est $L$-inférieur à $B$, et on notera $A \leq_{L} B$, si et seulement si $L(A) \leq L(B)$. Il est intéressant de remarquer que la condition $(* *)$ sur la forme linéaire $L$ donne alors un ordre total sur $\mathbb{N}^{s}$.

Pour tout élément $f$ de $\mathbb{K}[[X]]$, on définit aussi l'exposant privilégié de $f$ pour la direction $L$, ou $L$-ordre de $f$, le multi-indice $E=\operatorname{ord}_{L}(f)$ tel que

$$
f_{E} \neq 0 \quad \text { et } \quad f_{A}=0 \text { pour tout } A \in \mathbb{N}^{s} \text { avec } L(A)<L(E) .
$$

On a donc aussi

$$
\operatorname{ord}_{L}(f)=\min _{L}\left(J \in \mathbb{N}^{s}: J \in \operatorname{Exp}_{X}(f)\right) .
$$

4.1.1. ThÉorème (de $L$-division formelle [1]). Soient $f_{1}, \ldots, f_{p}$ des éléments de $\mathbb{K}[[X]]$. Pour tout $i=1, \ldots, p$ on note $E_{i}$ l'exposant privilégié dans la direction $L$ de $f_{i}$. Alors, pour tout $g \in \mathbb{K}[[X]]$, il existe des séries $g_{1}, \ldots, g_{p}, h$ de $\mathbb{K}[[X]]$ telles que

(i) $g=\sum_{i=1}^{p} f_{i} g_{i}+h$,

(ii) $\operatorname{Exp}_{X}\left(g_{i}(X) X^{E_{i}}\right) \subset \Delta_{i}, i=1, \ldots, p$,

(iii) $h=\sum_{b=0}^{\infty} \sum_{B \in \mathbb{N}^{s},|B|=b} h_{B} X^{B}$ avec $h_{B}=0$ pour $B \in \Delta$,

où $\mathbb{N}^{s}=\Delta_{1} \cup \ldots \cup \Delta_{p} \cup\left(\mathbb{N}^{s}-\Delta\right)$ est la partition de $\mathbb{N}^{s}$ associée aux $E_{i}, 1 \leq i \leq p$. De plus, $\left(g_{1}, \ldots, g_{p} ; h\right)$ est donné par un opérateur linéaire continu. En outre, si $\left(g_{1}^{\prime}, \ldots, g_{p}^{\prime} ; h^{\prime}\right)$ est un $p+1$-uplet satisfaisant (i)-(iii), alors on $a$, pour tout $i=1, \ldots, p, g_{i}^{\prime}=g_{i}$ et $h=h^{\prime}$.

Preuve. La démonstration de ce théorème peut être lue dans [1]. On peut, comme pour la proposition 1.6, en donner une preuve par perturbation d'un épimorphisme. On reprend les notations du paragraphe 1.1. On note alors

$$
(\mathbb{K}[[X]])^{E_{i}}=\left\{f \in \mathbb{K}[[X]]: \operatorname{Exp}_{X}\left(f(X) X^{E_{i}}\right) \subset \Delta_{i}\right\}
$$




$$
R_{\Delta}=\left\{f \in \mathbb{K}[[X]]: f(X)=\sum_{b=0}^{\infty} \sum_{\substack{B \in \mathbb{N}^{s} \\|B|=b}} f_{B} X^{B} \text { avec } f_{B}=0 \text { pour tout } B \in \Delta\right\} .
$$

On considère alors l'espace produit

$$
H_{\Delta}=\underset{i=1}{\stackrel{p}{\times}}(\mathbb{K}[[X]])^{E_{i}} \times R_{\Delta}
$$

que l'on munit de la valeur absolue $|\cdot|^{\Delta}$ définie par

$$
\left|\left(g_{1}, \ldots, g_{p}, h\right)\right|^{\Delta}=\sup _{i=1, \ldots, p}\left(e^{-L\left(E_{i}\right)-L\left(\operatorname{ord}_{L}\left(g_{i}\right)\right)}, e^{-L\left(\operatorname{ord}_{L}(h)\right)}\right) .
$$

On conclut alors comme dans [7]. L'unicité annoncée est élémentaire compte tenu des conditions (ii) et (iii).

On a facilement le résultat suivant.

4.1.2. Lemme. Soient $f$ et $g$ deux séries formelles non nulles. Alors, on $a$

(i) $\operatorname{ord}_{L}(f g)=\operatorname{ord}_{L}(f)+\operatorname{ord}_{L}(g)$ et

(ii) $\operatorname{ord}_{L}(f+g) \geq_{L} \min _{L}\left(\operatorname{ord}_{L}(f)\right.$, $\left.\operatorname{ord}_{L}(g)\right)$ si $f+g \neq 0$,

avec égalité dans (ii) si $\operatorname{ord}_{L}(f) \neq \operatorname{ord}_{L}(g)$.

4.2. Notion de base standard. Étant donné un idéal $\mathcal{I}$ de $\mathbb{K}[[X]]$, on note $E_{L}(\mathcal{I})$ l'ensemble des exposants privilégiés pour la direction $L$ des éléments de $\mathcal{I}$. On vérifie facilement que $A \in E_{L}(\mathcal{I})$ implique $A+\mathbb{N}^{s} \subset E_{L}(\mathcal{I})$.

On a aussi les résultats suivants.

4.2.1. LEMme. Si $h=\sum_{J \in \mathbb{N}^{s}, J \notin E_{L}(\mathcal{I})} h_{J} X^{J}$ appartient à $\mathcal{I}$, alors $h$ est identiquement nul.

On appelle base standard de $\mathcal{I}$ dans la direction $L$ une famille $\left(g_{1}, \ldots, g_{k}\right)$ de $\mathcal{I}$ telle que $E_{L}(\mathcal{I})=\bigcup_{i=1}^{k}\left(E_{i}+\mathbb{N}^{s}\right)$, où on note $E_{i}=\operatorname{ord}_{L}\left(g_{i}\right)$ pour tout $i=1, \ldots, k$.

On appelle frontière distinguée de $E_{L}(\mathcal{I})$ la plus petite partie $F_{L}(\mathcal{I})$ telle que $E_{L}(\mathcal{I})=\bigcup_{E \in F_{L}(\mathcal{I})}\left(E+\mathbb{N}^{s}\right)$. De manière évidente, $F_{L}(\mathcal{I})$ est une partie finie.

4.2.2. Lemme. Soit $\left(g_{1}, \ldots, g_{k}\right)$ une base standard de $\mathcal{I}$ dans la direction $L$. Si on note, pour tout $i=1, \ldots, k, E_{i}$ l'exposant privilégié de $g_{i}$ dans la direction $L$, alors $\mathcal{I}$ est engendré par l'ensemble des $\left(g_{j}\right)_{E_{j} \in F_{L}(\mathcal{I})}$.

On appelle alors l'ensemble $\left(g_{j}\right)_{E_{j} \in F_{L}(\mathcal{I})}$ base standard minimale de $\mathcal{I}$ dans la direction $L$.

Preuve. Soit $g$ une série formelle appartenant à l'idéal $\mathcal{I}$. On effectue, d'après le théorème 4.1.1, la $L$-division formelle de $g$ par l'ensemble des 
$\left(g_{j}\right)_{E_{j} \in F_{L}(\mathcal{I})}$. On obtient l'existence de séries formelles $h_{j}$ et $r$ telles que

$$
\operatorname{Exp}_{X}\left(h_{j}(X) X^{E_{j}}\right) \subset \Delta_{j}, \quad \operatorname{Exp}_{X}(r(X)) \subset \mathbb{N}^{s}-\Delta
$$

et

$$
g=\sum_{j: E_{j} \in F_{L}(\mathcal{I})} g_{j} h_{j}+r
$$

Par hypothèse, on a donc $r=g-\sum_{j: E_{j} \in F_{L}(\mathcal{I})} g_{j} h_{j} \in \mathcal{I}$, et le lemme 4.2.1 nous assure donc que $r=0$. Ceci achève la preuve du lemme.

Étant donné un idéal $\mathcal{I}$ de $\mathbb{K}[[X]]$ engendré par $p$ éléments $\left(f_{1}, \ldots, f_{p}\right)$ de $\mathbb{K}[[X]](M)$, on se propose de construire une base standard minimale $\left(g_{1}, \ldots, g_{k}\right)$ de $\mathcal{I}$ dans la direction $L$ telle que, pour tout $i=1, \ldots, k$, $g_{i}$ appartienne à $\mathbb{K}[[X]](M)$. On s'inspire de la construction des bases de Groebner pour les idéaux de polynômes donnée dans [4].

4.3. Définitions et notations. Soient $f$ et $g$ deux séries formelles non identiquement nulles. On note

$$
\begin{aligned}
f_{\operatorname{ord}_{L}(f)} & =\mathrm{FC}(f) & & (\text { le premier coefficient de } f), \\
X^{\operatorname{ord}_{L}(f)} & =\mathrm{FM}(f) & & (\text { le premier monôme de } f), \\
\mathrm{FC}(f) \cdot \operatorname{FM}(f) & =\mathrm{FT}(f) & & (\text { le premier terme de } f) .
\end{aligned}
$$

Si $\operatorname{ord}_{L}(f)=\alpha$ et $\operatorname{ord}_{L}(g)=\beta$, on pose $\gamma=\left(\gamma_{1}, \ldots, \gamma_{s}\right)$ avec $\gamma_{i}=$ $\max \left(\alpha_{i}, \beta_{i}\right)$ pour tout $i=1, \ldots, s$. On appelle alors $X^{\gamma}$ le plus petit commun multiple de $\operatorname{FM}(f)$ et $\operatorname{FM}(g)$ et on note $X^{\gamma}=\operatorname{PPCM}(\operatorname{FM}(f), \operatorname{FM}(g))$. On appelle $S$-série de $f$ et $g$ la combinaison

$$
S(f, g)=\frac{X^{\gamma}}{\mathrm{FT}(f)} f-\frac{X^{\gamma}}{\mathrm{FT}(g)} g .
$$

Cette combinaison est celle qui fait disparaître $\mathrm{FT}(f)$ et $\mathrm{FT}(g)$. Dans l'autre sens, on va voir dans le lemme suivant que, si on a une combinaison de séries dont les premiers termes s'annulent entre eux, celle-ci peut s'écrire comme combinaison de $S$-séries.

4.4. Lemme. Soient $g_{1}, \ldots, g_{t}, t$ séries formelles. On note $\alpha(1), \ldots, \alpha(t)$ $t$ multi-indices tels que $\alpha(i)+\operatorname{ord}_{L}\left(g_{i}\right)=\mu \in \mathbb{N}^{s}$. Soient $c_{1}, \ldots, c_{t}$ des constantes telles que, si on pose $f=\sum_{i=1}^{t} c_{i} X^{\alpha(i)} g_{i}$, ord $\operatorname{or}_{L}(f)$ est L-strictement supérieur à $\mu$. Il existe alors des constantes $c_{j, k}$ telles que l'on ait

$$
f=\sum_{j, k} c_{j, k} X^{\mu-\gamma_{j, k}} S\left(g_{j}, g_{k}\right),
$$

où $X^{\gamma_{j, k}}=\operatorname{PPCM}\left(\operatorname{FM}\left(g_{j}\right), \operatorname{FM}\left(g_{k}\right)\right)$. En outre, pour tout couple d'indices $(j, k)$, la série $X^{\mu-\gamma_{j, k}} S\left(g_{j}, g_{k}\right)$ a un L-ordre L-strictement supérieur à $\mu$. 
Preuve. On pose $d_{i}=\mathrm{FC}\left(g_{i}\right)$. Pour tout $i=1, \ldots, t, \operatorname{ord}_{L}\left(c_{i} X^{\alpha(i)} g_{i}\right)$ $=\mu$ et $\operatorname{ord}_{L}\left(\sum_{i=1}^{t} c_{i} X^{\alpha(i)} g_{i}\right)>_{L} \mu$. Cela implique alors

$$
\sum_{i=1}^{t} c_{i} d_{i}=0 .
$$

On pose $p_{i}=X^{\alpha(i)} g_{i} / d_{i}$. On remarque que l'on a $\mathrm{FC}\left(p_{i}\right)=1$ et aussi

$$
\begin{aligned}
f= & \sum_{i=1}^{t} c_{i} X^{\alpha(i)} g_{i}=\sum_{i=1}^{t} c_{i} d_{i} p_{i} \\
= & c_{1} d_{1}\left(p_{1}-p_{2}\right)+\left(c_{1} d_{1}+c_{2} d_{2}\right)\left(p_{2}-p_{3}\right)+\ldots \\
& +\left(c_{1} d_{1}+\ldots+c_{t-1} d_{t-1}\right)\left(p_{t-1}-p_{t}\right)+\left(c_{1} d_{1}+\ldots+c_{t} d_{t}\right) p_{t} .
\end{aligned}
$$

On pose $\mathrm{FT}\left(g_{i}\right)=d_{i} X^{\beta(i)}$. Alors, par hypothèse, pour tout $i=1, \ldots, t$, on a $\alpha(i)+\beta(i)=\mu$. Ainsi, pour tout $i=1, \ldots, t, \operatorname{FM}\left(g_{i}\right)=X^{\beta(i)}$ divise $X^{\mu}$. Par construction, on peut affirmer que, pour tout $j, k$ avec $j \neq k$, $X^{\gamma_{j, k}}=\operatorname{PPCM}\left(\operatorname{FM}\left(g_{j}\right), \operatorname{FM}\left(g_{k}\right)\right)$ divise $X^{\mu}$. Ainsi, $X^{\mu-\gamma_{j, k}}$ est un monôme et on a

$$
\begin{aligned}
X^{\mu-\gamma_{j, k}} S\left(g_{j}, g_{k}\right) & =X^{\mu-\gamma_{j, k}}\left(\frac{X^{\gamma_{j, k}}}{\operatorname{FT}\left(g_{j}\right)} g_{j}-\frac{X^{\gamma_{j, k}}}{\operatorname{FT}\left(g_{k}\right)} g_{k}\right) \\
& =\frac{X^{\mu}}{d_{j} X^{\beta(j)}} g_{j}-\frac{X^{\mu}}{d_{k} X^{\beta(k)}} g_{k} \\
& =\frac{X^{\alpha(j)}}{d_{j}} g_{j}-\frac{X^{\alpha(k)}}{d_{k}} g_{k}=p_{j}-p_{k} .
\end{aligned}
$$

En utilisant (4.4.1) et (4.4.2), $f$ peut être mise sous la forme

$$
\begin{aligned}
f= & \sum_{i=1}^{t} c_{i} X^{\alpha(i)} g_{i} \\
= & c_{1} d_{1} X^{\mu-\gamma_{1,2}} S\left(g_{1}, g_{2}\right)+\left(c_{1} d_{1}+c_{2} d_{2}\right) X^{\mu-\gamma_{2,3}} S\left(g_{2}, g_{3}\right)+\ldots \\
& \quad+\left(c_{1} d_{1}+\ldots+c_{t-1} d_{t-1}\right) X^{\mu-\gamma_{t-1, t}} S\left(g_{t-1}, g_{t}\right) .
\end{aligned}
$$

En outre, par hypothèse pour tout $i=1, \ldots, t$, le $L$-ordre de $p_{i}$ est exactement égal à $\mu$ et le premier coefficient vaut 1 ; ainsi, on a $\operatorname{ord}_{L}\left(p_{j}-p_{k}\right)>_{L} \mu$. L'égalité (4.4.3) nous permet alors d'affirmer que $\operatorname{ord}_{L}\left(X^{\mu-\gamma_{j, k}} S\left(g_{j}, g_{k}\right)\right)$ $>_{L} \mu$.

Le critère suivant, analogue à celui de détermination des bases de Groebner pour des idéaux de polynômes [4], va permettre de vérifier si une famille de $\mathcal{I}$ est une base standard.

4.5. Lemme. Soit $\mathcal{I}$ un idéal de $\mathbb{K}[[X]]$. Soit $L$ une forme linéaire vérifiant $(* *)$. Une famille $G=\left(g_{1}, \ldots, g_{t}\right)$ est une base standard pour $\mathcal{I}$ dans 
la direction $L$ si et seulement si, pour tout couple d'indices $(i, j)$ avec $i \neq j$, le reste de la L-division formelle de $S\left(g_{i}, g_{j}\right)$ par $G$ est nul.

Preuve. Le sens direct est élémentaire. En effet, $S\left(g_{i}, g_{j}\right)$, pour tout couple d'indices $(i, j)$, appartient à $\mathcal{I}$. Puisque $G=\left(g_{1}, \ldots, g_{t}\right)$ est une base standard pour $\mathcal{I}$ dans la direction $L$, le lemme 4.2.1 permet alors d'affirmer que le reste de la $L$-division formelle de $S\left(g_{i}, g_{j}\right)$ par $G$ est nul. Pour établir la réciproque, considérons une série formelle $f \in \mathcal{I}$ non nulle. Il existe donc des séries $\left(h_{1}, \ldots, h_{t}\right) \in \mathbb{K}[[X]]$ telles que l'on ait

$$
f=\sum_{i=1}^{t} h_{i} g_{i} .
$$

Soit $m_{h}(i)=\operatorname{ord}_{L}\left(h_{i} g_{i}\right)$. On définit $\mu_{h}=\min _{L}\left(m_{h}(1), \ldots, m_{h}(t)\right)$. On a clairement

$$
\operatorname{ord}_{L}(f) \geq_{L} \mu_{h} .
$$

On considère l'ensemble des écritures possibles de $f$ sous la forme (4.5.1). Comme l'ordre considéré est total, on choisit une expression de la forme (4.5.1),

$$
f=\sum_{i=1}^{t} H_{i} g_{i},
$$

telle que $\mu_{H}$ soit maximale, c'est-à-dire $\mu_{H} \geq_{L} \mu_{h}$ pour tout ensemble $h=$ $\left(h_{1}, \ldots, h_{t}\right)$ tel que $f$ s'écrive sous la forme (4.5.1). Pour alléger les notations, on pose $\mu=\mu_{H}$. Nous allons montrer que $\operatorname{ord}_{L}(f)=\mu$.

On suppose

$$
\operatorname{ord}_{L}(f)>_{L} \mu .
$$

On isole dans l'expression (4.5.1) choisie pour $f$ les termes d'ordre $\mu$. On peut donc écrire, en notant $\mu=\min _{L}(m(1), \ldots, m(t))$,

$$
\begin{aligned}
f & =\sum_{m(i)=\mu} H_{i} g_{i}+\sum_{m(i)>_{L} \mu} H_{i} g_{i} \\
& =\sum_{m(i)=\mu} \mathrm{FT}\left(H_{i}\right) g_{i}+\sum_{m(i)=\mu}\left(H_{i}-\mathrm{FT}\left(H_{i}\right)\right) g_{i}+\sum_{m(i)>_{L} \mu} H_{i} g_{i} .
\end{aligned}
$$

Les monômes qui apparaissent dans la seconde et la troisième somme ont tous un $L$-ordre $L$-strictement supérieur à $\mu$. L'hypothèse $\operatorname{ord}_{L}(f)>_{L} \mu$ nous assure que la première somme a aussi un $L$-ordre $L$-strictement supérieur à $\mu$. On pose $\mathrm{FT}\left(H_{i}\right)=c_{i} X^{\alpha(i)}$. On a donc

$$
\sum_{m(i)=\mu} \mathrm{FT}\left(H_{i}\right) g_{i}=\sum_{m(i)=\mu} c_{i} X^{\alpha(i)} g_{i} .
$$


Les hypothèses du lemme 4.4 sont vérifiées; cela implique donc l'existence de constantes $c_{j, k}$ telles que l'on ait

$$
\sum_{m(i)=\mu} \mathrm{FT}\left(H_{i}\right) g_{i}=\sum_{j, k} c_{j, k} X^{\mu-\gamma_{j, k}} S\left(g_{j}, g_{k}\right)
$$

et

$$
\operatorname{ord}_{L}\left(X^{\mu-\gamma_{j, k}} S\left(g_{j}, g_{k}\right)\right)>_{L} \mu,
$$

où $X^{\gamma_{j, k}}=\operatorname{PPCM}\left(\operatorname{FM}\left(g_{j}\right), \operatorname{FM}\left(g_{k}\right)\right)$. Puisque, par hypothèse, le reste de la $L$-division formelle de $S\left(g_{j}, g_{k}\right)$ par $G$ est nul, on peut écrire

$$
S\left(g_{j}, g_{k}\right)=\sum_{i=1}^{t} a_{i j k} g_{i},
$$

où $a_{i j k} \in \mathbb{K}[[X]]$ et $\operatorname{ord}_{L}\left(a_{i j k} g_{i}\right) \geq_{L} \operatorname{ord}_{L}\left(S\left(g_{j}, g_{k}\right)\right)$ pour tout $i, j, k$. En effet, pour tout $i=1, \ldots, p, \operatorname{ord}_{L}\left(a_{i j k} g_{i}\right) \subset \Delta_{i}$ avec $\Delta_{i} \cap \Delta_{l}=\emptyset$ pour tout $l \neq i$.

Ainsi, l'égalité (4.5.7) donne

$$
\operatorname{ord}_{L}\left(S\left(g_{j}, g_{k}\right)\right)=\min _{i}\left(\operatorname{ord}_{L}\left(a_{i j k} g_{i}\right)\right)
$$

et

$$
X^{\mu-\gamma_{j, k}} S\left(g_{j}, g_{k}\right)=\sum_{i=1}^{t} b_{i j k} g_{i},
$$

où $b_{i j k}=a_{i j k} X^{\mu-\gamma_{j, k}}$. De plus, (4.5.8) et (4.5.6) impliquent

$$
\operatorname{ord}_{L}\left(b_{i j k} g_{i}\right) \geq_{L} \operatorname{ord}_{L}\left(X^{\mu-\gamma_{j, k}} S\left(g_{j}, g_{k}\right)\right)>_{L} \mu .
$$

En outre, en utilisant (4.5.5) et (4.5.9), on obtient

$$
\begin{aligned}
\sum_{m(i)=\mu} \mathrm{FT}\left(H_{i}\right) g_{i} & =\sum_{j, k} c_{j, k} X^{\mu-\gamma_{j, k}} S\left(g_{j}, g_{k}\right) \\
& =\sum_{j, k} c_{j, k}\left(\sum_{i} b_{i j k} g_{i}\right)=\sum_{i}\left(\sum_{j, k} c_{j, k} b_{i j k}\right) g_{i} .
\end{aligned}
$$

On pose $\sum_{j, k} c_{j, k} b_{i j k}=\widetilde{H}_{i}$. L'inégalité (4.5.10) implique alors, pour tout $i$,

$$
\operatorname{ord}_{L}\left(\widetilde{H}_{i} g_{i}\right)>_{L} \mu \text {. }
$$

On remplace dans (4.5.3) $\sum_{m(i)=\mu} \mathrm{FT}\left(H_{i}\right) g_{i}$ par $\sum_{i} \widetilde{H}_{i} g_{i}$ pour obtenir

$$
f=\sum_{i} \widetilde{H}_{i} g_{i}+\sum_{m(i)=\mu}\left(H_{i}-\mathrm{FT}\left(H_{i}\right)\right) g_{i}+\sum_{m(i)>_{L} \mu} H_{i} g_{i} .
$$

On a donc trouvé une expression de $f$ du type (4.5.1) où tous les termes ont un $L$-ordre $L$-strictement supérieur à $\mu$. Ceci est impossible par maximalité de $\mu$. L'hypothèse $\operatorname{ord}_{L}(f)>_{L} \mu$ est donc absurde. On conclut 
aisément avec (4.5.2) que $\operatorname{ord}_{L}(f)=\mu$. On a donc bien $\operatorname{FM}(f)=X^{\mu}$ avec $\mu=\min _{L}(m(1), \ldots, m(t))$. Ainsi $\mu$ appartient à $E_{L}(\mathcal{I})$ et $G$ est une base standard de $\mathcal{I}$ pour la direction $L$.

On considère l'ordre naturel défini, pour $I=\left(i_{1}, \ldots, i_{s}\right)$ et $J=\left(j_{1}, \ldots, j_{s}\right)$, par

$$
\begin{aligned}
& I \leq_{\text {nat }} J \quad \text { équivaut à } \\
& \quad I=J \text {, ou }|I|<|J| \text {, ou } \\
& \quad|I|=|J| \text { et } \exists k \text { tel que } i_{l}=j_{l} \text { pour } l=1, \ldots, k-1, \text { et } i_{k}>j_{k} .
\end{aligned}
$$

Soient $I, J$ des multi-indices de $\mathbb{N}^{s}$. Si $I \leq_{\text {nat }} J$ et $I \neq J$, on écrira $I<_{\text {nat }} J$. On peut considérer pour cet ordre l'exposant privilégié d'un élément $f$ de $\mathbb{K}[[X]]$. De plus, on montre aisément que, étant donné un entier $k$, il existe une forme linéaire $L$ vérifiant (**) qui préserve l'ordre naturel jusqu'à l'entier $k$, c'est-à-dire telle que, pour tous multi-indices $I, J \in \mathbb{N}^{s}$ satisfaisant $|I| \leq k$ et $|J| \leq k$, on ait

$$
I<_{\text {nat }} J \Rightarrow L(I)<L(J) .
$$

4.6. Proposition. Soient $f_{1}, \ldots, f_{p}$ des éléments de $\mathbb{K}[[X]](M)$. On note $\mathcal{I}$ l'idéal engendré par ces éléments sur $\mathbb{K}[[X]]$. Il existe alors une forme linéaire $L$ vérifiant $(* *)$ et une base standard minimale $G=\left(g_{1}, \ldots, g_{k}\right)$ pour $\mathcal{I}$ dans la direction $L$ telle que, pour tout $i=1, \ldots, k, g_{i}$ appartient $\grave{a}$ $\mathbb{K}[[X]](M)$.

Preuve. La preuve de cette proposition donne en plus une construction explicite de cette base standard.

On pose $G_{0}=\left(f_{1}, \ldots, f_{p}\right)$. Soit alors, pour tout $i=1, \ldots, p, E_{i}^{(0)}$ l'exposant privilégié, pour l'ordre naturel, associé à $f_{i}$. Soit $k_{0}$ un entier tel que $k_{0}>\max _{i=1, \ldots, p}\left|E_{i}^{(0)}\right|$. Soit $L_{0}$ une forme linéaire vérifiant (**) qui préserve l'ordre naturel jusqu'à l'entier $k_{0}$. Les multi-indices $E_{i}^{(0)}$ sont aussi les exposants privilégiés dans la direction $L_{0}$ des séries $f_{i}$. On peut s'assurer que $G_{0}$ est $\left(E_{1}^{(0)}, \ldots, E_{p}^{(0)} ; L_{0}\right)$-régulière dans $\mathbb{K}[[X]]$. On se trouve dans les conditions du théorème 2.4. Pour chaque couple d'indices $(i, j)$ avec $i \neq j$, on ajoute alors à $G_{0}$ l'ensemble des restes non nuls de la $L_{0}$-division des séries $S\left(f_{i}, f_{j}\right)$ par $G_{0}$. On appelle $G_{1}$ l'ensemble ainsi construit. Si $G_{1}=G_{0}$, d'après le lemme 4.5, $G_{0}$ est une base standard de $\mathcal{I}$ dans la direction $L_{0}$. Sinon, on note $r_{i, j}^{(1)}$ le reste de la $L_{0}$-division de $S\left(f_{i}, f_{j}\right)$ par $G_{0}$ et $E_{i, j}^{(1)}$ l'exposant privilégié de $r_{i, j}^{(1)}$ pour l'ordre naturel. Clairement $r_{i, j}^{(1)}$ appartient à l'idéal $\mathcal{I}$.

Si $\max _{i, j}\left|E_{i, j}^{(1)}\right|<k_{0}$, ces multi-indices sont aussi les exposants privilégiés dans la direction $L_{0}$. Sinon, soit $k_{1}>\max _{i, j}\left|E_{i, j}^{(1)}\right|$; alors on peut trouver $L_{1}$ une forme linéaire qui préserve l'ordre naturel usuel jusqu'à 
l'entier $k_{1}$. Alors, $L_{1}$ est un "raffinement" de $L_{0}$ en ce sens que les restes issus de la $L_{1}$-division des $S$-séries de $G_{0}$ coïncident avec les restes issus de la $L_{0}$-division des $S$-séries de $G_{0}$, puisque les exposants privilégiés dans la direction $L_{1}$ de $G_{0}$ sont les mêmes que ceux dans la direction $L_{0}$.

On réitère alors le processus ; c'est-à-dire, pour chaque couple d'indices $(i, j)$, avec $i \neq j$, on ajoute à $G_{1}$ l'ensemble des restes non nuls de la division des séries $S\left(f_{i}, f_{j}\right)$ par $G_{1}$. On appelle $G_{2}$ l'ensemble ainsi construit. Si $G_{2}=$ $G_{1}$, d'après le lemme $4.5, G_{1}$ est une base standard de $\mathcal{I}$ dans la direction $L_{1}$. Sinon, on recommence. L'algorithme s'arrête lorsque l'on trouve $i \in \mathbb{N}$ tel que $G_{i+1}=G_{i}$; alors, $G_{i}$ est une base standard pour $\mathcal{I}$ dans la direction $L_{i}$. En outre, toutes les séries qui composent les ensembles $G_{k}$ sont issus de restes de la $L_{i}$-division d'une série de $\mathbb{K}[[X]](M)$ par des séries de $\mathbb{K}[[X]](M)$, ce sont donc aussi, d'après le théorème 2.4, des séries qui appartiennent à $\mathbb{K}[[X]](M)$.

Pour achever la preuve, il reste à montrer que l'algorithme comporte un nombre fini d'itérations, c'est-à-dire qu'il existe bien un entier $i$ tel que pour tout entier $k \geq i, G_{k}=G_{i}$. Pour cela, on considère l'idéal $\left\langle\mathrm{FT}\left(G_{k}\right)\right\rangle$ engendré dans $\mathbb{K}[X]$ par l'ensemble des $\mathrm{FT}(h)$ pour tout $h \in G_{k}$. Si $G_{i+1} \neq G_{i}$, on peut affirmer que $\left\langle\mathrm{FT}\left(G_{i}\right)\right\rangle$ est strictement plus petit que $\left\langle\mathrm{FT}\left(G_{i+1}\right)\right\rangle$. En effet, par hypothèse, au moins un reste non nul $r$ a été ajouté à $G_{i}$. Comme $r$ est un reste de division par l'idéal engendré par $G_{i}, \mathrm{FT}(r)$ n'est divisible par aucun des $\mathrm{FT}(h), h \in G_{i}$. On a donc une suite croissante d'idéaux monômiaux dans l'anneau des polynômes $\mathbb{K}[X]$ qui est noethérien; elle est donc stationnaire à partir d'un certain rang. Ceci achève la démonstration.

On a donc obtenu une base standard de $\mathcal{I}$ dans la direction de la forme linéaire $L=L_{i}$. Pour avoir une base standard minimale, il suffit d'appliquer le lemme 4.2.2, c'est-à-dire de ne garder que les éléments de $G_{i}$ dont les exposants privilégiés dans la direction $L_{i}$ appartiennent à la frontière distinguée de $\mathcal{I}$ dans la direction $L_{i}$.

4.7. Étude d'un exemple. On considère l'idéal $\mathcal{I}$ sur $\mathbb{K}[[X, Y]]$ engendré par

$$
f_{1}(X, Y)=X^{2}+Y^{3}+\sum_{n=4}^{\infty} M_{n} Y^{n} \quad \text { et } \quad f_{2}(X, Y)=X+Y .
$$

On a donc clairement $f_{i} \in \mathbb{K}[[X, Y]](M)$ pour $i=1,2$. Nous allons construire une base standard minimale de $\mathcal{I}$ suivant un ordre proche de l'ordre naturel. Pour cela, on applique l'algorithme donné dans la proposition 4.6.

On a $G_{0}=\left(f_{1}, f_{2}\right), E_{1}^{(0)}=(2,0), E_{2}^{(0)}=(1,0)$. En choisissant alors $L_{0}\left(i_{1}, i_{2}\right)=i_{1}+\sqrt{2} i_{2}, L_{0}$ vérifie bien $(* *)$ et les exposants privilégiés de $f_{1}$ et $f_{2}$ dans la direction de $L_{0}$ sont respectivement $E_{1}^{(0)}$ et $E_{2}^{(0)}$. En outre, 
on a

$$
S\left(f_{1}, f_{2}\right)(X, Y)=Y^{3}-X Y+\sum_{n=4}^{\infty} M_{n} Y^{n} .
$$

On effectue alors la $L_{0}$-division de $S\left(f_{1}, f_{2}\right)$ par $G_{0}$. On obtient

$$
\begin{aligned}
S\left(f_{1}, f_{2}\right)(X, Y) & =-(X+Y) Y+\left(Y^{2}+Y^{3}+\sum_{n=4}^{\infty} M_{n} Y^{n}\right) \\
& =-Y f_{1}(X, Y)+Y^{2}+Y^{3}+\sum_{n=4}^{\infty} M_{n} Y^{n} .
\end{aligned}
$$

Ainsi, en posant $f_{3}(X, Y)=Y^{2}+Y^{3}+\sum_{n=4}^{\infty} M_{n} Y^{n}$, on a

$$
G_{1}=\left(f_{1}(X, Y), f_{2}(X, Y), f_{3}(X, Y)\right),
$$

et, suivant l'ordre naturel, les exposants privilégiés de $f_{1}, f_{2}$ et $f_{3}$ sont respectivement $E_{1}^{(1)}=(2,0), E_{2}^{(1)}=(1,0), E_{3}^{(1)}=(0,2)$. Ils coïncident bien avec les exposants privilégiés dans la direction de $L_{0}$. Ainsi, on pose $L_{1}=L_{0}$. En outre, on a

$$
\begin{aligned}
& S\left(f_{1}, f_{2}\right)(X, Y)=Y^{3}-X Y+\sum_{n=4}^{\infty} M_{n} Y^{n}, \\
& S\left(f_{1}, f_{3}\right)(X, Y)=Y^{5}-X^{2} Y^{3} \\
& +\sum_{n=4}^{\infty} M_{n} Y^{n+2}-X^{2} Y^{3}-\sum_{n=4}^{\infty} M_{n} X^{2} Y^{n}, \\
& \text { (4.7.6) } S\left(f_{2}, f_{3}\right)(X, Y)=Y^{3}-X Y^{3}-\sum_{n=4}^{\infty} M_{n} X Y^{n} \text {. }
\end{aligned}
$$

On effectue alors la $L_{1}$-division des $S$-séries $S\left(f_{i}, f_{j}\right)$, pour $i, j=1,2,3$, $i \neq j$, par $G_{1}$. On obtient

$$
\begin{aligned}
S\left(f_{1}, f_{2}\right)(X, Y)= & -Y f_{1}(X, Y)+f_{3}(X, Y) \\
S\left(f_{1}, f_{3}\right)(X, Y)= & -\left(Y^{3}+\sum_{n=4}^{\infty} M_{n} Y^{n}\right) f_{1}(X, Y) \\
& +\left(Y^{3}+\sum_{n=4}^{\infty} M_{n} Y^{n}\right) f_{3}(X, Y) . \\
S\left(f_{2}, f_{3}\right)(X, Y)= & -\left(Y^{3}+\sum_{n=4}^{\infty} M_{n} Y^{n}\right) f_{2}(X, Y)+Y f_{3}(X, Y) .
\end{aligned}
$$

L'ensemble des restes de ces divisions sont nuls, ainsi, d'après la preuve de la proposition $4.6, G_{1}=\left(f_{1}, f_{2}, f_{3}\right)$ est une base standard de $\mathcal{I}$ suivant la 
direction $L_{1}$. On remarque alors que, pour $i=1,2,3, f_{i}$ appartient bien à $\mathbb{K}[[X, Y]](M)$. Enfin, à partir de $G_{1}$, on a facilement une base standard minimale de $\mathcal{I}$, en remarquant que

$$
\left(E_{1}+\mathbb{N}^{2}\right) \cup\left(E_{2}+\mathbb{N}^{2}\right) \cup\left(E_{3}+\mathbb{N}^{2}\right)=\left(E_{2}+\mathbb{N}^{2}\right) \cup\left(E_{3}+\mathbb{N}^{2}\right) .
$$

Ainsi, $\left(f_{2}(X, Y), f_{3}(X, Y)\right)=\left(X+Y, Y^{2}+Y^{3}+\sum_{n=4}^{\infty} M_{n} Y^{n}\right)$ est une base standard minimale de $\mathcal{I}$ suivant la direction $L_{1}$. On vérifie d'ailleurs que

$$
X^{2}+Y^{3}+\sum_{n=4}^{\infty} M_{n} Y^{n}=\left(Y^{2}+Y^{3}+\sum_{n=4}^{\infty} M_{n} Y^{n}\right)+(X-Y)(X+Y) .
$$

On a alors le théorème, annoncé en 0.3 , de division par un idéal pour des séries appartenant à des classes de séries formelles à croissance contrôlée et son corollaire 4.9.

4.8. ThÉorÈme. Soient $f_{1}, \ldots, f_{p}$ des éléments de $\mathbb{K}[[X]](M)$. On note $\mathcal{I}$ l'idéal engendré par ces éléments sur $\mathbb{K}[[X]]$. Il existe alors une forme linéaire $L$ vérifiant $(* *)$ et une base standard $G=\left(g_{1}, \ldots, g_{k}\right)$ pour $\mathcal{I}$ dans la direction $L$ telle que, pour tout $i=1, \ldots, k, g_{i}$ appartienne $\mathbb{K}[[X]](M)$, et telle que, pour tout $g$ dans $\mathbb{K}[[X]](M)$, on puisse écrire $g=\sum_{i=1}^{k} h_{i} g_{i}+h_{0}$ avec $h_{i}$ dans $\mathbb{K}[[X]](M)$ pour tout $i=0, \ldots, k$. En outre, $g$ appartient à $\mathcal{I}$ si et seulement si $h_{0}=0$.

Preuve. C'est une conséquence du théorème 2.4 et de la proposition 4.6. On note que, d'après la définition de la base standard et l'allure du reste après division (voir (1.4.1)), si $g$ appartient à $\mathcal{I}$, nécessairement on a $h_{0}=0$. Ceci n'est pas toujours vérifié si $G$ n'est pas une base standard pour $\mathcal{I}$ dans la direction $L$ (voir (4.10.4)).

4.9. Corollaire. Soit $\mathcal{I}$ un idéal de $\mathbb{K}[[X]](M)$. Il existe alors une forme linéaire $L$ vérifiant $(* *)$ et une base standard minimale $\left(g^{E}\right)_{E \in F_{L}(\mathcal{I})}$ de $\mathcal{I}$, telles que l'on ait

$\mathcal{I}$ est engendré par $\left(g^{E}\right)_{E \in F_{L}(\mathcal{I})}$, tout élément $g \in \mathbb{K}[[X]](M)$ est équivalent modulo $\mathcal{I}$ à un unique élément $h$ de $\mathbb{K}[[X]](M)$ de la forme $h=\sum_{J \notin E_{L}(\mathcal{I})} h_{J} X^{J}$.

$L$ 'anneau $\mathbb{K}[[X]](M)$ est donc noethérien.

Preuve. Ce corollaire est une conséquence immédiate du théorème 4.8 et du lemme 4.2.2. En utilisant le fait que la frontière distinguée $F_{L}(\mathcal{I})$ de l'ensemble $E_{L}(\mathcal{I})$ des exposants privilégiés de $\mathcal{I}$ dans la direction $L$ est une partie finie de $\mathbb{N}^{s}$, on retrouve alors la noethérianité de l'anneau $\mathbb{K}[[X]](M)$.

On donne dans la suite un exemple de l'importance du choix de la famille génératrice de l'idéal lors de la division par cet idéal. 
4.10. Étude d'un exemple. On considère l'idéal $\mathcal{I}$ sur $\mathbb{K}[[X, Y]]$ engendré par

$$
f_{1}(X, Y)=X^{2}+Y^{3} \quad \text { et } f_{2}(X, Y)=X^{5} .
$$

Soit $M_{n}=n$ !. Soit $\left(u_{n}\right)_{n \in \mathbb{N}}$ une suite de réels tels que

$$
u_{0}=0, \quad u_{p}=0 \quad \text { pour tout entier } p \not \equiv 0[6] \quad \text { et } \quad u_{6 n}=M_{6 n} .
$$

On pose $g(X, Y)=\sum_{n=1}^{\infty} u_{2 n} X^{n} Y^{n}$. On a donc clairement $g \in \mathbb{K}[[X, Y]](M)$. En outre, on vérifie que l'on a

$$
\begin{aligned}
g(X, Y)= & \left(X^{2}+Y^{3}\right)\left(\sum_{k=1}^{\infty} \sum_{n=0}^{\infty}(-1)^{k-1} u_{2 n+6 k} X^{n+5 k-2} Y^{n}\right) \\
& +X^{5}\left(\sum_{k=1}^{\infty}(-1)^{k} u_{6 k} X^{5(k-1)}\right) .
\end{aligned}
$$

On a donc écrit $g$ sous la forme

$$
g(X, Y)=q_{1}(X, Y) f_{1}(X, Y)+q_{2}(X, Y) f_{2}(X, Y),
$$

ainsi $g \in \mathcal{I}$. Mais, $q_{1}(X, Y)$ s'écrit

$$
\begin{aligned}
q_{1}(X, Y)= & \sum_{k=1}^{\infty}(-1)^{k-1} u_{6 k} X^{5 k-2} \\
& +\sum_{k=1}^{\infty} \sum_{n=1}^{\infty}(-1)^{k-1} u_{2 n+6 k} X^{n+5 k-2} Y^{n} \\
= & q_{1}^{(1)}(X, Y)+q_{1}^{(2)}(X, Y) .
\end{aligned}
$$

Il est clair que la série $q_{1}^{(1)}(X, Y)$ n'appartient pas à $\mathbb{K}[[X, Y]](M)$. Par conséquent, puisque les séries $q_{1}^{(1)}(X, Y)$ et $q_{1}^{(2)}(X, Y)$ ont un support disjoint, on déduit de (4.10.3) que la série $q_{1}(X, Y)$ n'appartient pas à $\mathbb{K}[[X, Y]](M)$. On vérifie de même que la série $q_{2}(X, Y)$ n'appartient pas, non plus, à $\mathbb{K}[[X, Y]](M)$. Ainsi, pour $i=1,2, q_{i}$ n'appartient pas à $\mathbb{K}[[X, Y]](M)$.

Cependant, si on applique directement le théorème 2.4, avec une forme linéaire $L$ qui associe aux séries $f_{1}(X, Y)$ et $f_{2}(X, Y)$ les mêmes exposants privilégiés que ceux donnés par l'ordre naturel (on peut prendre $L\left(i_{1}, i_{2}\right)=$ $i_{1}+\sqrt{2} i_{2}$ ), on trouve

$$
\begin{aligned}
g(X, Y)= & \left(X^{2}+Y^{3}\right)\left(\sum_{k=1}^{\infty} \sum_{n=0}^{\infty}(-1)^{k-1} u_{2 n+4 k} X^{n} Y^{n+5 k-3}\right) \\
& +\sum_{k=0}^{\infty}(-1)^{k}\left(u_{4 k} Y^{5 k}+u_{4 k+2} X Y^{5 k+1}\right) .
\end{aligned}
$$

Ainsi, on vérifie, en utilisant l'hypothèse $\left(\mathrm{H}_{2}\right)$ sur la suite $\left\{M_{n}\right\}_{n \in \mathbb{N}}$, que le 
quotient et le reste appartiennent bien à $\mathbb{K}[[X, Y]](M)$. Cependant, bien que $g$ appartienne à $\mathcal{I}$, le reste de cette division n'est pas nul.

Le problème se pose donc de construire un système fini de générateurs $\left(g_{i}\right)$ de l'idéal $\mathcal{I}$ appartenant à $\mathbb{K}[[X, Y]](M)$ tel que l'on puisse écrire, pour tout $u \in \mathcal{I}$,

$$
u(X, Y)=\sum_{i} h_{i}^{(u)} g_{i}
$$

avec $h_{i}^{(u)} \in \mathbb{K}[[X, Y]](M)$ pour tout $i$.

Selon le schéma de la preuve de la proposition 4.6, on construit alors une base standard minimale de $\mathcal{I}$ dans la direction $L$. On obtient

$$
\left(g_{1}(X, Y), g_{2}(X, Y), g_{3}(X, Y)\right)=\left(X^{2}+Y^{3}, X Y^{6}, Y^{9}\right) .
$$

Et par rapport à cette nouvelle base, on applique le théorème 2.4 pour obtenir

$$
\begin{aligned}
g(X, Y)= & \left(X^{2}+Y^{3}\right)\left(\sum_{k=1}^{\infty} \sum_{n=0}^{\infty}(-1)^{k-1} u_{2 n+4 k} X^{n} Y^{n+5 k-3}\right) \\
& +X Y^{6}\left(\sum_{k=1}^{\infty}(-1)^{k} u_{4 k+2} Y^{5(k-1)}\right) \\
& +Y^{9}\left(\sum_{k=2}^{\infty}(-1)^{k} u_{4 k} Y^{5 k-9}\right) .
\end{aligned}
$$

Cette fois, on a bien écrit $g$ sous la forme (4.10.5) avec $h_{i}^{(g)} \in \mathbb{K}[[X, Y]](M)$.

4.11. Relations de division. Soient $f_{1}, \ldots, f_{p}$ des éléments de $\mathbb{K}[[X]](M)$. On note $\mathcal{I}$ l'idéal engendré par ces éléments sur $\mathbb{K}[[X]]$. Le théorème 4.8 assure qu'il existe $g_{1}, \ldots, g_{k}$, dans $\mathbb{K}[[X]](M)$, des générateurs de $\mathcal{I}$ tels que, pour toute série $g$ de $\mathbb{K}[[X]](M)$ appartenant à $\mathcal{I}$, la division de $g$ par $G=\left(g_{1}, \ldots, g_{k}\right)$ donne

$$
g=\sum_{i=1}^{k} h_{i} g_{i}
$$

avec $h_{i} \in \mathbb{K}[[X]](M)$ pour tout $i=1, \ldots, k$. Il est alors naturel de se poser la question suivante : peut-on écrire $g$ sous la forme $g=\sum_{i=1}^{p} \widetilde{h}_{i} f_{i}$ avec $\widetilde{h}_{i} \in$ $\mathbb{K}[[X]](M)$ pour tout $i=1, \ldots, p$ ? La division 2.4 appliquée directement à $g$ ne donne pas forcément un reste nul (voir 4.10). En fait, en utilisant à nouveau la construction de la proposition 4.6, on a une réponse affirmative à la question.

ThÉorème (Relations dans $\mathbb{K}[[X]](M)$ ). Soient $f_{1}, \ldots, f_{p}$ des éléments de $\mathbb{K}[[X]](M)$. On note $\mathcal{I}$ l'idéal engendré par ces éléments sur $\mathbb{K}[[X]]$. Alors, pour toute série $g$ de $\mathbb{K}[[X]](M)$ appartenant à $\mathcal{I}$, on peut écrire 
$g=\sum_{i=1}^{p} q_{i} f_{i}$ avec $q_{i}$ dans $\mathbb{K}[[X]](M)$ pour tout $i=1, \ldots, p$. Si on note $\mathcal{I}_{M}$ l'idéal engendré par $f_{1}, \ldots, f_{p}$ sur $\mathbb{K}[[X]](M)$, on a donc

$$
\mathcal{I}_{M}=\mathcal{I} \cap \mathbb{K}[[X]](M) .
$$

Preuve. C'est une conséquence directe de la construction opérée dans la preuve de la proposition 4.6. En effet, le théorème 4.8 assure qu'il existe une direction $L$ et une base standard $g_{1}, \ldots, g_{k}$ de $\mathcal{I}$ dans cette direction telles que

$$
g=\sum_{i=1}^{k} h_{i} g_{i}
$$

avec $h_{i} \in \mathbb{K}[[X]](M)$ pour tout $i=1, \ldots, k$. On regarde alors comment cette base standard est construite. A la première étape, on ajoute, pour une forme linéaire $L$ bien choisie, les restes $r_{i, j}$ non nuls de la $L$-division des $S$-séries $S\left(f_{i}, f_{j}\right)$, pour $i, j=1, \ldots, p, i \neq j$, par $\left(f_{1}, \ldots, f_{p}\right)$. On a donc

$$
S\left(f_{i}, f_{j}\right)=\sum_{k=1}^{p} q_{k}^{(i, j)} f_{k}+r_{i, j}
$$

avec, pour tout $i, j=1, \ldots, p, i \neq j$, et pour tout $k=1, \ldots, p, q_{k}^{(i, j)}$ et $r_{i, j}$ des séries de $\mathbb{K}[[X]](M)$. Or, par construction, on a

$$
S\left(f_{i}, f_{j}\right)=\frac{X^{\gamma_{i, j}}}{\mathrm{FT}\left(f_{i}\right)} f_{i}-\frac{X^{\gamma_{i, j}}}{\mathrm{FT}\left(f_{j}\right)} f_{j} .
$$

On peut donc écrire, pour tout $i, j=1, \ldots, p, i \neq j$,

$$
r_{i, j}=\sum_{k=1}^{p} \widetilde{q}_{k}^{(i, j)} f_{k}
$$

avec, pour tout $i, j=1, \ldots, p, i \neq j$, et pour tout $k=1, \ldots, p, \widetilde{q}_{k}^{(i, j)}$ des séries de $\mathbb{K}[[X]](M)$. On réitère ce processus à chaque étape de construction de la base standard pour conclure que, pour tout $i=1, \ldots, k$, on peut écrire

$$
g_{i}=\sum_{l=1}^{p} \widetilde{h}_{i} f_{i}
$$

avec $h_{i}$ dans $\mathbb{K}[[X]](M)$ pour tout $i=1, \ldots, p$. On utilise alors (4.11.1) pour obtenir le résultat escompté.

4.12. Retour à l'exemple précédent. On considère l'idéal $\mathcal{I}$ sur $\mathbb{K}[[X, Y]]$ engendré par

$$
f_{1}(X, Y)=X^{2}+Y^{3} \quad \text { et } f_{2}(X, Y)=X^{5} .
$$

On reprend la série $g(X, Y)$. On sait qu'elle appartient à l'idéal $\mathcal{I}$. On veut donc écrire $g(X, Y)=h_{1}(X, Y) f_{1}(X, Y)+h_{2}(X, Y) f_{2}(X, Y)$ avec $h_{1}(X, Y)$ 
et $h_{2}(X, Y)$ des séries de $\mathbb{K}[[X, Y]](M)$. La base standard minimale de $\mathcal{I}$ construite dans (4.10.6) est $\left(X^{2}+Y^{3}, X Y^{6}, Y^{9}\right)$. En effet, la première étape de la construction donne

$$
S\left(f_{1}, f_{2}\right)=X^{3} Y^{3}=X^{3}\left(X^{2}+Y^{3}\right)-X^{5} .
$$

La division de $S\left(f_{1}, f_{2}\right)$ par $\left(f_{1}, f_{2}\right)$ donne

$$
X^{3}\left(X^{2}+Y^{3}\right)-X^{5}=X Y^{3}\left(X^{2}+Y^{3}\right)-X Y^{6} .
$$

On ajoute alors à $\left(f_{1}(X, Y), f_{2}(X, Y)\right)$ le reste non nul de cette division, c'est-à-dire $X Y^{6}$. On obtient l'ensemble

$$
E_{1}=\left(X^{2}+Y^{3}, X^{5}, X Y^{6}\right)
$$

avec

$$
X Y^{6}=\left(-X^{3}+X Y^{3}\right)\left(X^{2}+Y^{3}\right)+X^{5} .
$$

De même, à $E_{1}$ on ajoute les restes non nuls de la division des $S$-séries de $E_{1}$ par $E_{1}$. On obtient simplement

$$
S\left(X^{2}+Y^{3}, X Y^{6}\right)=Y^{6}\left(X^{2}+Y^{3}\right)-X\left(X Y^{6}\right)=Y^{9} .
$$

Ainsi, on a, en regroupant (4.12.4) et (4.12.5),

$$
Y^{9}=\left(Y^{6}+X^{4}-X^{2} Y^{3}\right)\left(X^{2}+Y^{3}\right)-(X) X^{5} .
$$

Finalement, en remplaçant dans (4.10.8) $X Y^{6}$ par son expression issue de (4.12.4) et $Y^{9}$ par son expression issue de (4.12.6), on obtient

$$
\begin{aligned}
= & \left(X^{2}+Y^{3}\right)\left(\sum_{k=1}^{\infty} \sum_{n=0}^{\infty}(-1)^{k-1} u_{2 n+4 k} X^{n} Y^{n+5 k-3}\right) \\
& +\left(\left(-X^{3}+X Y^{3}\right)\left(X^{2}+Y^{3}\right)+X^{5}\right)\left(\sum_{k=1}^{\infty}(-1)^{k} u_{4 k+2} Y^{5(k-1)}\right) \\
& +\left(\left(Y^{6}+X^{4}-X^{2} Y^{3}\right)\left(X^{2}+Y^{3}\right)-(X) X^{5}\right)\left(\sum_{k=2}^{\infty}(-1)^{k} u_{4 k} Y^{5 k-9}\right) .
\end{aligned}
$$

On voit que l'on peut écrire $g(X, Y)=\left(X^{2}+Y^{3}\right) h_{1}(X, Y)+X^{5} h_{2}(X, Y)$ avec $h_{1}(X, Y)$ et $h_{2}(X, Y)$ des séries de $\mathbb{K}[[X, Y]](M)$.

\section{Références}

[1] J. M. Aroca, H. Hironaka and J. L. Vicente, The theory of the maximal contact, Memorias de Matematica del Instituto "Jorge Juan", 29 Madrid, 1975.

[2] J. Briançon, Weierstrass préparé à la Hironaka, Singularités à Cargèse, Astérisque 7-8 (1973), 67-73. 
[3] J. Chaumat et A.-M. Chollet, Caractérisation des anneaux noethériens de séries formelles à croissance contrôlée. Application à la synthèse spectrale, Publ. Mat. 41 (1997), 545-561.

[4] D. Cox, J. Little and D. O'Shea, Ideals, Varieties and Algorithms, Springer, 1992.

[5] H. Grauert und R. Remmert, Analytische Stellenalgebren, Grundlehren Math. Wiss. 176, Springer, Berlin, 1971.

[6] A. Mouze, Un théorème d'Artin pour des anneaux de séries formelles à croissance contrôlée, C. R. Acad. Sci. Paris Sér. I Math. 330 (2000), 15-20.

[7] J. C. Tougeron, Idéaux de fonctions différentiables, Ergeb. Math. Genzgeb. 71, Springer, Berlin, 1972.

[8] M. A. Zurro, On the rings of formal solutions of polynomial differential equations, in: Banach Center Publ. 44, Inst. Math., Polish Acad. Sci., Warszawa, 1998, 277-292.

CNRS-UMR 8524

Mathématiques, bâtiment M2

Université des Sciences et Technologies de Lille

59655 Villeneuve d'Ascq Cedex, France

E-mail: mouze@agat.univ-lille1.fr

Received February 7, 2000

Revised version October 18, 2000 